\title{
A delay differential model of ENSO variability: parametric instability and the distribution of extremes
}

\author{
M. Ghil ${ }^{1,4}$, I. Zaliapin ${ }^{2}$, and S. Thompson ${ }^{3}$ \\ ${ }^{1}$ Dépt. Terre-Atmosphère-Océan and Laboratoire de Météorologie Dynamique, Ecole Normale Supérieure, Paris, France \\ ${ }^{2}$ Dept. of Mathematics and Statistics, University of Nevada, Reno, NV, USA \\ ${ }^{3}$ Dept. of Mathematics and Statistics, University of Radford, VA, USA \\ ${ }^{4}$ Dept. of Atmospheric and Oceanic Sciences and Institute of Geophysics and Planetary Physics, University of California Los \\ Angeles, CA, USA
}

Received: 17 October 2007 - Revised: 26 March 2008 - Accepted: 26 March 2008 - Published: 28 May 2008

\begin{abstract}
We consider a delay differential equation (DDE) model for El-Niño Southern Oscillation (ENSO) variability. The model combines two key mechanisms that participate in ENSO dynamics: delayed negative feedback and seasonal forcing. We perform stability analyses of the model in the three-dimensional space of its physically relevant parameters. Our results illustrate the role of these three parameters: strength of seasonal forcing $b$, atmosphere-ocean coupling $\kappa$, and propagation period $\tau$ of oceanic waves across the Tropical Pacific. Two regimes of variability, stable and unstable, are separated by a sharp neutral curve in the $(b, \tau)$ plane at constant $\kappa$. The detailed structure of the neutral curve becomes very irregular and possibly fractal, while individual trajectories within the unstable region become highly complex and possibly chaotic, as the atmosphere-ocean coupling $\kappa$ increases. In the unstable regime, spontaneous transitions occur in the mean "temperature" (i.e., thermocline depth), period, and extreme annual values, for purely periodic, seasonal forcing. The model reproduces the Devil's bleachers characterizing other ENSO models, such as nonlinear, coupled systems of partial differential equations; some of the features of this behavior have been documented in general circulation models, as well as in observations. We expect, therefore, similar behavior in much more detailed and realistic models, where it is harder to describe its causes as completely.
\end{abstract}

\section{Introduction and motivation}

\subsection{Key ingredients of ENSO theory}

The El-Niño/Southern-Oscillation (ENSO) phenomenon is the most prominent signal of seasonal-to-interannual climate

Correspondence to: M. Ghil

(ghil@atmos.ucla.edu) variability. It was known for centuries to fishermen along the west coast of South America, who witnessed a seemingly sporadic and abrupt warming of the cold, nutrient-rich waters that support the food chain in those regions; these warmings caused havoc to their fish harvests (Diaz and Markgraf, 1992; Philander, 1990). The common occurrence of such warming shortly after Christmas inspired them to name it El Niño, after the "Christ child." Starting in the 1970s, researchers found that El Niño's climatic effects were far broader than just its manifestations off the shores of Peru (Diaz and Markgraf, 1992; Glantz et al., 1991). This realization led to a global awareness of ENSO's significance, and an impetus to attempt and improve predictions of exceptionally strong El Niño events (Latif et al., 1994).

The following conceptual elements have been shown to play a determining role in the dynamics of the ENSO phenomenon.

(i) The Bjerknes hypothesis: Bjerknes (1969), who laid the foundation of modern ENSO research, suggested a positive feedback as a mechanism for the growth of an internal instability that could produce large positive anomalies of sea surface temperatures (SSTs) in the eastern Tropical Pacific. We use here the climatological meaning of the term anomaly, i.e., the difference between an instantaneous (or short-term average) value and the normal (or long-term mean). Using observations from the International Geophysical Year (1957-1958), Bjerknes realized that this mechanism must involve air-sea interaction in the tropics. The "chain reaction" starts with an initial warming of SSTs in the "cold tongue" that occupies the eastern part of the equatorial Pacific. This warming causes a weakening of the thermally direct Walkercell circulation; this circulation involves air rising over the warmer SSTs near Indonesia and sinking over the colder SSTs near Peru. As the trade winds blowing from the east weaken and thus give way to westerly wind anomalies, the ensuing local changes in the ocean circulation encourage further SST increase.

Published by Copernicus Publications on behalf of the European Geosciences Union and the American Geophysical Union. 
Thus the feedback loop is closed and further amplification of the instability is triggered.

(ii) Delayed oceanic wave adjustments: Compensating for Bjerknes's positive feedback is a negative feedback in the system that allows a return to colder conditions in the basin's eastern part (Suarez and Schopf, 1988). During the peak of the cold-tongue warming, called the warm or El Niño phase of ENSO, westerly wind anomalies prevail in the central part of the basin. As part of the ocean's adjustment to this atmospheric forcing, a Kelvin wave is set up in the tropical wave guide and carries a warming signal eastward; this signal deepens the eastern-basin thermocline, which separates the warmer, well-mixed surface waters from the colder waters below, and thus contributes to the positive feedback described above. Concurrently, slower Rossby waves propagate westward, and are reflected at the basin's western boundary, giving rise therewith to an eastward-propagating Kelvin wave that has a cooling, thermocline-shoaling effect. Over time, the arrival of this signal erodes the warm event, ultimately causing a switch to a cold, La Niña phase.

(iii) Seasonal forcing: A growing body of work (Ghil and Robertson, 2000; Chang et al., 1994, 1995; Jin et al., 1994, 1996; Tziperman et al., 1994, 1995) points to resonances between the Pacific basin's intrinsic air-sea oscillator and the annual cycle as a possible cause for the tendency of warm events to peak in boreal winter, as well as for ENSO's intriguing mix of temporal regularities and irregularities. The mechanisms by which this interaction takes place are numerous and intricate and their relative importance is not yet fully understood (Tziperman et al., 1995; Battisti, 1988).

\subsection{Formulation of DDE models}

Starting in the 1980s, the effects of delayed feedbacks and external forcing have been studied using the formalism of delay differential equations (DDE); see, inter alia, Bhattacharya et al. (1982) and Ghil and Childress (1987) for geoscience applications, and Hale (1977) and Nussbaum (1998) for DDE theory.

Several DDE systems have been suggested as toy models for ENSO variability. Battisti and Hirst (1989) have considered the linear autonomous DDE

$d T / d t=-\alpha T(t-\tau)+T, \quad \alpha>0, \tau>0$.

Here, $T$ represents the sea-surface temperature (SST) averaged over the eastern equatorial Pacific. The first term on the right-hand side (rhs) of Eq. (1) mimics the negative feedback due to the oceanic waves, while the second term reflects Bjerknes's positive feedback. As shown in (Battisti and Hirst, 1989), Eq. (1) reproduces some of the main features of a fully nonlinear coupled atmosphere-ocean model of ENSO dynamics in the tropics (Battisti, 1988; Zebiak and Cane, 1987).
Suarez and Schopf (1988) and Battisti and Hirst (1989) also studied a nonlinear version of Eq. (1), in which a cubic nonlinearity is added to the rhs of the equation:

$d T / d t=-\alpha T(t-\tau)+T-T^{3}$,

where $0<\alpha<1$ and $\tau>0$. This system has three steady states, obtained by finding the roots of the rhs:

$T_{0}=0, \quad T_{1,2}= \pm(1-\alpha)^{1 / 2}$.

The so-called inner solution $T_{0}$ is always unstable, while the outer solutions $T_{1,2}$ may be stable or unstable depending on the parameters $(\alpha, \tau)$. If an outer steady state is unstable, the system exhibits bounded oscillatory dynamics; in (Suarez and Schopf, 1988) it was shown numerically that a typical period of such oscillatory solutions is about two times the delay $\tau$.

The delay equation idea was very successful in explaining the periodic nature of ENSO events. Indeed, the delayed negative feedback does not let a solution fade away or blow up, as in the ordinary differential equation (ODE) case with $\tau=0$, and thus creates an internal oscillator with period depending on the delay and particular form of the equation's rhs. DDE modeling has also emphasized the importance of nonlinear interactions in shaping the complex dynamics of the ENSO cycle. At the same time, many important details of ENSO variability still had to be explained.

First, a delayed oscillator similar to Eq. (1) or Eq. (2) typically has periodic solutions with well-defined periods. However, the occurrence of ENSO events is irregular and can only be approximated very coarsely by a periodic function. Second, El-Niño events always peak during the Northern Hemisphere (boreal) winter, hence their name; such phase locking cannot be explained by a purely internal delayed oscillator. Third, the value of the period produced by the delay equations deviates significantly from actual ENSO interevent times of 2-7 years. The delay $\tau$, which is the sum of the basin-transit times of the westward Rossby and eastward Kelvin waves, can be roughly estimated to lie in the range of 6-8 months. Accordingly, model Eq. (2) suggests a period of 1.5-2 years, at most, for the repeating warm events; this is about half the dominant ENSO recurrence time.

Tziperman et al. (1994) have demonstrated that these discrepancies can be removed - still within the DDE framework - by considering nonlinear interactions between the internal oscillator and the external periodic forcing by the seasonal cycle. These authors also introduced a more realistic type of nonlinear coupling between atmosphere and ocean to reflect the fact that the delayed negative feedback saturates as the absolute value of the key dependent variable $T$ increases; note that in Eq. (1) the feedback is linearly proportional to the delayed state variable $T(t-\tau)$. Munnich et al. (1991) studied an iterated-map model of ENSO and made a detailed comparison between cubic and sigmoid nonlinearities. 
As a result, the sigmoid type of nonlinearity was chosen in (Tziperman et al., 1994), resulting in the periodically forced, nonlinear DDE

$$
\begin{aligned}
d T / d t & =-\alpha \tanh \left[\kappa T\left(t-\tau_{1}\right)\right] \\
& +\beta \tanh \left[\kappa T\left(t-\tau_{2}\right)\right]+\gamma \cos (2 \pi t) .
\end{aligned}
$$

Model Eq. (3) was shown to have solutions that possess an integer period, are quasiperiodic, or exhibit chaotic behavior, depending on the parameter values. The increase of solution complexity - from period one, to integer but higher period, and on - to quasiperiodicity and chaos - is caused by the increase of the atmosphere-ocean coupling parameter $\kappa$. The study (Tziperman et al., 1994) also demonstrated that this forced DDE system exhibits period locking, when the external, "explicit" oscillator wins the competition with the internal, delayed one, causing the system to stick to an integer period; see also the more detailed analysis of phase locking in the intermediate coupled model (ICM) of Jin et al. (1994; 1996).

To summarize our motivation for the choice of a "toy model," work during the past 30 years has shown that ENSO dynamics is governed, by and large, by the interplay of several nonlinear mechanisms that can be studied in simple forced DDE models. Such models provide a convenient paradigm for explaining interannual ENSO variability and shed new light on its dynamical properties. So far, though, DDE model studies of ENSO have been limited to linear stability analysis of steady-state solutions, which are not typical in forced systems, case studies of particular trajectories, or one-dimensional scenarios of transition to chaos, varying a single parameter while the others are kept fixed. A major obstacle for the complete bifurcation and sensitivity analysis of such DDE models lies in the complex nature of DDEs, whose numerical and analytical treatment is much harder than that of their ODE counterparts.

In this work we take several steps toward a comprehensive analysis of DDE models relevant for ENSO phenomenology. In doing so, we also wish to illustrate the complexity of phase-parameter space structure for even such a simple model of climate dynamics.

Specifically, we study the behavior of model solutions in a broad three-dimensional domain of its physically relevant parameters. In this domain, we find and illustrate several regions of stable and unstable solution behavior; some of these regions have a complex and possibly fractal geometric structure, and we examine the distribution of essential solution properties throughout this domain in parameter space. One of the most important characteristics of a solution is the value of its extrema, i.e., its global maximum or minimum; these extrema are directly connected to the strength of a corresponding El-Niño or La-Niña event. We only focus in the present paper on the dependence of the global extrema on the model parameters; the equally interesting issue of the distribution of the values and occurrence times of local extrema will be reported elsewhere.
In Sect. 2, we formulate our DDE model, provide basic theoretical results for this type of DDEs, present the numerical integration method used, and describe several solution types and their possible physical interpretation. In Sect. 3, we proceed to explore fully solution behavior over a broad range of the model's three most physically relevant parameters. We reproduce several dynamical solution features and bifurcation scenarios previously reported in the literature for both simpler (Saunders and Ghil, 2001) and more detailed (Jin et al., 1994, 1996; Tziperman et al., 1994, 1995; Ghil and Robertson, 2000; Neelin et al., 1994, 1998; Dijkstra, 2005) models, report new ones, and describe the corresponding three-dimensional (3-D) regime diagram. This 3-D regime diagram includes large regions of very smooth parameter dependence, as well as regions of very sensitive dependence on the parameters; the neutral surface separating simpler from more complex behavior exhibits rich and apparently fractal patterns.

The discussion in Sect. 4 highlights the possibility of spontaneous, intrinsic transitions between the presence or absence of intraseasonal, higher-frequency fluctuations, as well as of interdecadal, lower-frequency variability. Such higher- and lower-frequency variability accompanies the seasonal and interannual oscillations that dominate our model solutions. This coexistence of variabilities on several time scales affects not only the mean and period of the solutions, but also the distribution of extreme warm and cold events.

An illustration of the complexity "burst" caused by introducing a scalar delay in a simple ODE is given in Appendix A. Appendix B contains the proof of a key theoretical result presented in Sect. 2, while Appendix C provides details on numerical procedures for DDEs.

\section{Model and numerical integration method}

\subsection{Model formulation and parameters}

We consider a nonlinear DDE with additive, periodic forcing,

$\frac{d h(t)}{d t}=-a \tanh [\kappa h(t-\tau)]+b \cos (2 \pi \omega t) ;$

here $t \geq 0$ and the parameters $a, \kappa, \tau, b$, and $\omega$ are all real and positive. Equation (4) is a simplified one-delay version of the two-delay model considered by Tziperman et al. (1994); it mimics two mechanisms essential for ENSO variability: delayed negative feedback via the function $\tanh (\kappa z)$ and periodic external forcing. As we shall see, these two mechanisms suffice to generate very rich behavior that includes several important features of more detailed models and of observational data sets. Including the positive Bjerkness feedback (Philander, 1990; Bjerknes, 1969; Neelin et al., 1994, 1998) is left for future work.

The function $h(t)$ in Eq. (4) represents the thermocline depth deviations from the annual mean in the Eastern 
Pacific; accordingly, it can also be roughly interpreted as the regional SST, since a deeper thermocline corresponds to less upwelling of cold waters, and hence higher SST, and vice versa. The thermocline depth is affected by the wind-forced, eastward Kelvin and westward Rossby oceanic waves. The waves' delayed effects are modeled by the function $\tanh [\kappa h(t-\tau)]$; the delay $\tau$ is due to the finite wave velocity and corresponds roughly to the combined basin-transit time of the Kelvin and Rossby waves.

The particular form of the delayed nonlinearity plays a very important role in the behavior of a DDE model. Munnich et al. (1991) provide a physical justification for the monotone, sigmoid nonlinearity we adopt. The parameter $\kappa$, which is the linear slope of $\tanh (\kappa z)$ at the origin, reflects the strength of the atmosphere-ocean coupling. The forcing term represents the seasonal cycle in the trade winds.

The model Eq. (4) is fully determined by its five parameters: feedback delay $\tau$, atmosphere-ocean coupling strength $\kappa$, feedback amplitude $a$, forcing frequency $\omega$, and forcing amplitude $b$. By an appropriate rescaling of time $t$ and dependent variable $h$, we let $\omega=1$ and $a=1$. The other three parameters may vary, reflecting different physical conditions of ENSO evolution. We consider here the following ranges of these parameters: $0 \leq \tau \leq 2 \mathrm{yr}, 0<\kappa<\infty, 0 \leq b<\infty$.

To completely specify the DDE model Eq. (4) we need to prescribe some initial "history," i.e. the behavior of $h(t)$ on the interval $[-\tau, 0)$ (Hale, 1977). In most of the numerical experiments below we assume $h(t) \equiv 1,-\tau \leq t<0$, i.e. we start with a warm year. Numerical experiments with alternative specifications of the initial history suggest that this choice does not affect our qualitative conclusions.

\subsection{Basic theoretical results}

To develop some intuition about the dynamics of Eq. (4), we consider two limiting cases. In the absence of the feedback, $a=0$, the model becomes a simple ODE and hence has only a sinusoidal solution with period 1 . One expects to observe the same behavior for $b / a \gg 1$. In the absence of forcing, $b=0$, we obtain a well-studied DDE

$\dot{h}(t)=-g[h(t-\tau)], \quad g(z)=\tanh (\kappa z)$.

The character of the solutions of this equation depends strongly on the delay $\tau$. For small delays, one expects to see behavior reminiscent of the corresponding ODE with zero delay; the general validity of such "small-delay expectations" is analyzed in detail in (Bodnar, 2004). For larger delays the nonlinear feedback might produce more complex dynamics. These heuristic intuitions happen to be true: Eq. (5) has an asymptotic solution that is identically zero for $\tau \leq \tau_{0}$, and admits periodic solutions with period $4 \tau$ for $\tau>\tau_{0}$, where the critical delay is $\tau_{0}=\pi /(2 \kappa)$ (Cao, 1996; Nussbaum, 1979; Chow and Walter, 1988). In addition, it is known that the null solution is the only stable solution for $\tau \leq \tau_{0}$. At $\tau=\tau_{0}$ the system undergoes a Hopf bifurcation, and the trivial steady state transfers its stability to a periodic solution. Among other solutions, an important role is played by the so-called slow oscillating solutions, whose zeros are separated by a distance of at least the delay $\tau$. In particular, Chow and Walther (1988) showed that periodic solutions with period $4 \tau$ and the symmetry condition $-h(t)=h(t-2 \tau)$ are exponentially asymptotically stable; that is, any other solution will approach one of these solutions at an exponential rate. Moreover, for $\tau>\tau_{0}$, these solutions may be the only stable ones (Chow and Walter, 1988).

These results can help explain the observed nearperiodicity of ENSO variability. If one takes the delay $\tau$ to equal approximately the transit time of the traveling ocean waves, namely 6 to 8 months, then the $4 \tau$ internal period of the ENSO oscillator becomes $2-3$ years. This provides a good justification for the observed quasi-biennial oscillation in Tropical Pacific SSTs and trade winds (Diaz and Markgraf, 1992; Philander, 1990; Jiang et al., 1995; Ghil et al., 2002).

It should be emphasized though that in Eq. (5) we only retained the negative feedback associated with the westwardtraveling Rossby waves, and neglected the the positive feedback associated with the eastward-traveling Kelvin waves; see Tziperman et al. (1994). Cane et al. (1990) provide a detailed discussion of how the interplay between positive and negative feedbacks affects the ENSO interevent time (see their Summary and Discussion section, p. 1572). Additional evidence for the 2-3-year period in this ENSO model is provided by our further numerical results in Sect. 2.4 and Sect. 3.3, Figs. 6 and 7.

Below we summarize basic theoretical results about Eq. (4). Following the traditional framework (Hale, 1977; Nussbaum, 1998), we consider the Banach space $X=C([-\tau, 0), \mathbb{R})$ of continuous functions $h:[-\tau, 0) \rightarrow \mathbb{R}$ and define the norm for $h \in X$ as

$\|h\|=\sup \{|h(t)|, t \in[-\tau, 0)\}$,

where $|\cdot|$ denotes the absolute value in $\mathbb{R}$. For convenience, we reformulate the DDE initial-value problem (IVP) in its rescaled form:

$$
\begin{aligned}
\frac{d h(t)}{d t} & =-\tanh [\kappa h(t-\tau)]+b \cos (2 \pi t), t \geq 0, \\
h(t) & =\phi(t) \text { for } t \in[-\tau, 0), \quad \phi(t) \in X .
\end{aligned}
$$

Proposition 1 (Existence, uniqueness, continuous dependence) For any fixed positive triplet $(\tau, \kappa, b)$, the IVP Eqs. (6)-(7) has a unique solution $h(t)$ on $[0, \infty)$. This solution depends continuously on the initial data $\phi(t)$, delay $\tau$ and the rhs of Eq. (6) considered as a continuous map $f:[0, T) \times X \rightarrow \mathbb{R}$, for any finite $T$.

Proof. See Appendix B.

From Proposition 1 it follows, in particular, that the system Eqs. (6)-(7) has a unique solution for all time, which depends 
continuously on the model parameters $(\tau, \kappa, b)$ for any finite time. This result implies that any discontinuity in the solution profile as a function of the model parameters indicates the existence of an unstable solution that separates the attractor basins of two stable solutions.

Our numerical experiments suggest, furthermore, that all stable solutions of Eqs. (6)-(7) are bounded and have an infinite number of zeros.

\subsection{Numerical integration}

The results in this study are based on numerical integration of the DDE Eqs. (6)-(7). We emphasize that there are important differences between numerical integration of DDEs and ODEs. The first important difference is in the requisite initial data. The solution of a system of ODEs is determined by its value at the initial point $t=t_{0}$. When integrating a DDE, terms like $h(t-\tau)$ may represent values of the solution at points prior to the initial point. Because of this, the given initial data must include not only $h\left(t_{0}\right)$, but also a "history" of the values $h(t)$ for all $t$ prior to $t_{0}$ in the interval that extends as far back at the largest delay.

Another important issue in solving DDEs arises when a delayed value of the argument falls within the current integration step. In order to avoid limiting the step size to be smaller than the smallest delay, or, alternatively, to avoid extrapolating the previous solution, an iterative procedure must be used to obtain successive approximations of the delayed solution that will yield a satisfactory local error estimate. The implementation of this iterative procedure affects profoundly the performance of a DDE solver.

These and other specific features of DDE numerical integration require development of special software and often the problem-specific modification of such software. We used here the Fortran 90/95 DDE solver dde_solver of Shampine and Thompson (2006), available at http://www. radford.edu/ thompson/ffddes/. This solver implements a $(5,6)$ pair of continuously embedded, explicit Runge-KuttaSarafyan methods (Corwin et al., 1997). Technical details of dde_solver, as well as a brief overview of other available DDE solvers are given in Appendix C.

The numerical simulations in this paper require very long integration intervals, leading to prohibitive storage requirements. This difficulty led us to incorporate several new options in dde_solver; they are also described in Appendix C.

\subsection{Examples of model dynamics}

In this subsection we illustrate typical solutions of the problem Eqs. (6)-(7) and comment on physically relevant aspects of these solutions. All experiments shown here use the constant initial data $\phi \equiv 1$. Figure 1 shows six trajectories obtained by fixing $b=1, \kappa=100$ and varying the delay $\tau$ over two orders of magnitude, from $\tau=10^{-2}$ to $\tau=1$, with $\tau$ increasing from bottom to top in the figure. The sequence of

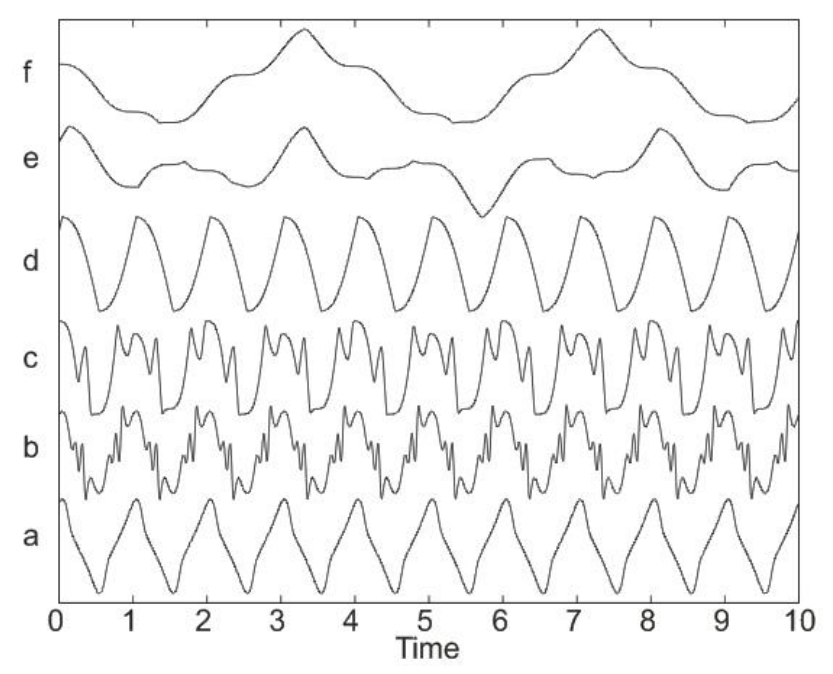

Fig. 1. Examples of DDE model solutions. Model parameters are $\kappa=100$ and $b=1$, while $\tau$ increases from curve (a) to curve (f) as follows: (a) $\tau=0.01$, (b) $\tau=0.025$, (c) $\tau=0.15$, (d) $\tau=0.45$, (e) $\tau=0.995$, and (f) $\tau=1$.

changes in solution type illustrated in Fig. 1 is typical for any choice of $(b, \kappa)$ as $\tau$ increases.

For a small delay, $\tau<\pi /(2 \kappa)$, we have a periodic solution with period 1 (curve a); here the internal oscillator is completely dominated by the seasonal forcing. When the delay increases, the effect of the internal oscillator becomes visible: small wiggles, in the form of amplitude-modulated oscillations with a period of $4 \tau$, emerge as the trajectory crosses the zero line. However, these wiggles do not affect the overall period, which is still unity. The wiggle amplitude grows with $\tau$ (curve b) and eventually wins over the seasonal oscillations, resulting in period doubling (curve c). Further increase of $\tau$ results in the model passing through a sequence of bifurcations that produce solution behavior of considerable interest for understanding ENSO variability.

Some of these types of solution behavior are illustrated further in Fig. 2. Panel (a) $(\kappa=5, \tau=0.65)$ shows the occurrence of "low- $h$," or cold, events every fourth seasonal cycle. Note that negative values of $h$ correspond to the boreal (Northern Hemisphere) winter, that is to the upwelling season - December-January-February - in the eastern Tropical Pacific; in the present, highly idealized model, we can associate the extreme negative values with large-amplitude cold events, or La Niñas. This solution pattern loses its regularity when the atmosphere-ocean coupling increases: Panel (b) ( $\kappa=100, \tau=0.58)$ shows irregular occurrence of large cold events with the interevent time varying from 3 to 7 cycles.

In panel (c) $(\kappa=50, \tau=0.42)$ we observe alternately and irregularly occurring warm El-Niño and cold La Niña events: the "high- $h$ " events occur with a period of about 4 years and random magnitude. Panel (d) $(\kappa=500, \tau=0.005)$ shows another interesting type of behavior: bursts of intraseasonal 
a)

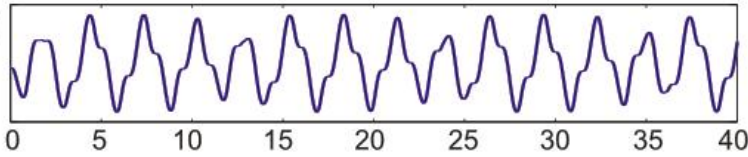

b)

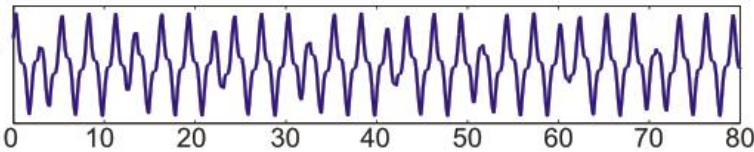

c)

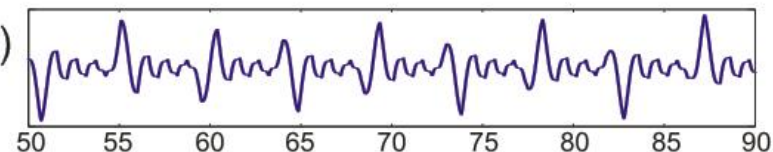

d)

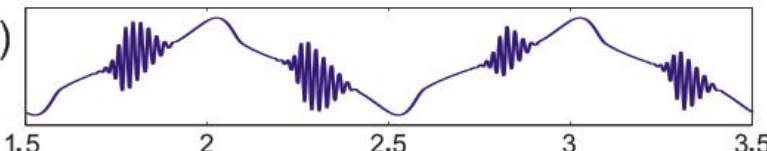

e)

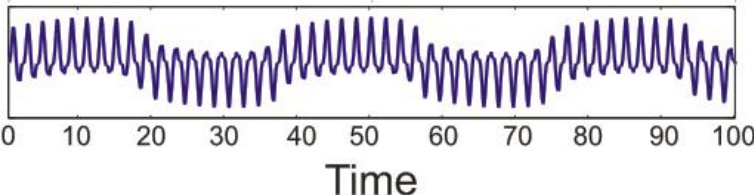

Fig. 2. Noteworthy solution patterns of relevance to ENSO dynamics; seasonal forcing amplitude $b=1$. (a)) Regularly occurring cold (low- $h$ ) events, or La Niñas $(\kappa=5, \tau=0.65)$; (b) irregular cold events $(\kappa=100, \tau=0.58) ;(\mathbf{c})$ irregular alternations of warm (El Niño, high- $h$ ) and cold events $(\kappa=50, \tau=0.42)$; (d) intraseasonal activity reminiscent of Madden-Julian oscillations or westerly-wind bursts ( $\kappa=500, \tau=0.005)$; and (e) interdecadal variability in the annual mean and in the relative amplitude of warm and cold events $(\kappa=50, \tau=0.508)$

oscillations of random amplitude superimposed on regular, period-one dynamics. This pattern is reminiscent of Madden-Julian oscillations (Madden and Julian, 1971, 1972, 1994) or westerly-wind bursts (Gebbie et al., 2007; Harrison and Giese, 1988; Verbickas, 1998; Delcroix et al., 1993; Boulanger et al., 2004; Lengaigne et al., 2004; Saynisch et al., 2006). Westerly wind bursts are physically related to atmospheric convection that is not a part of the current model. The somewhat surprising model result of high-frequency, intraseasonal variability suggests that realistic bursts might be excited in the atmosphere by or interact synergistically with the apparently slower mechanisms represented by this coupled model: they could be triggered by, rather than trigger, warm or cold ENSO episodes.

The solution in panel (e) $(\kappa=50, \tau=0.508)$ demonstrates sustained interdecadal variability in the absence of any external source of such variability. The solution pattern illustrates spontaneous changes in the long-term annual mean, as well as in the distribution of positive and negative extremes, with respect to both time and amplitude.

\section{Critical transitions}

\subsection{Numerical characterization of solution behavior}

In this section we focus on the onset of instabilities in the model Eqs. (6)-(7). Taking a "metric" approach to the problem, we study the change in several statistics of a trajectory as the model parameters change. This approach is complementary to the "topological" one, which forms the basis for the stability analysis of dynamical systems (Andronov and Pontryagin, 1937; Katok and Hasselblatt, 1995). In the latter, one studies the topological structure of the system's attractor(s), i.e. a combination of points, circles, tori, or more complicated objects. The motivation for this approach comes from noting that topologically equivalent solutions can be mapped onto each other using an appropriate diffeomorphism, i.e. a one-to-one, continuously differentiable map. Hence, considering topologically equivalent classes rather than all individual solutions is enough for studying the system's qualitative behavior. From a practical point of view, though, metric properties of a solution might be as important as its topological description or more: think of living in a region with constant air temperature of $-10^{\circ} \mathrm{C}$ vs. $20^{\circ} \mathrm{C}$. Furthermore, metric properties are also much easier to study, in simple models, as well as in full, 3-D general circulation models (GCMs) and in observational data sets.

Technically, we proceed in the following way. For each fixed triplet of parameters $(b, \kappa, \tau)$ we find a numerical approximation $\hat{h}_{i} \equiv \hat{h}\left(t_{i}\right)$ of the model solution $h(t)$ on a grid $\mathcal{G}=\left\{t_{i}\right\}_{i=1, \ldots, N}, t_{i} \in\left[0, T_{\max }\right]$ using the Fortran $90 \mathrm{DDE}$ solver dde_solver of Shampine and Thompson (2006) (see also Appendix C). We only consider the latter part of each solution, $t_{i}>T_{\min }>0$, in order to avoid any transient effects; to simplify notations, we assume from now on that $t_{1}=T_{\min }$. Typical parameters for our numerical experiments are $T_{\max }=10^{4}, T_{\max }-T_{\min }=10^{3}$, time step $\delta=t_{i}-t_{i-1}=10^{-3}$, and a numerical precision of $\epsilon=10^{-4}$. We have also verified some of the results with a precision up to $10^{-12}$, a time step of $10^{-4}$, and over time intervals up to $T_{\max }-T_{\min }=10^{4}$ in order to ascertain that the reported phenomena are not caused or affected by numerical errors.

We report results for the following trajectory statistics: maximum value $M=\max _{i} \hat{h}_{i}$; mean value $E=\sum_{i} \hat{h}_{i} / N$, where $N$ is the maximal integer less than $\left(T_{\max }-T_{\min }\right) / \delta$; and mean of positive values $E_{+}=\sum_{i} \hat{h}_{i} \mathbf{1}_{\hat{h}_{i}>0} / \sum_{i} \mathbf{1}_{\hat{h}_{i}>0}$, where $\mathbf{1}_{A}$ is the characteristic function of the set $A$, identically equal to one for all points in $A$ and zero outside of $A$. Furthermore, we have computed, but do not show here, the trajectory variance, the mean of negative values, and upper 90\% and 95\% quantiles; the results are very similar for all the statistics we have examined. 
We also computed an approximation to the period of a solution. Specifically, for any positive integer $\Delta$ we define the $\Delta$-discrepancy

$$
\begin{aligned}
R_{\Delta} & =\frac{\sum_{i=\Delta+1}^{N}\left(\hat{h}_{i}-\hat{h}_{i-\Delta}\right)^{2}}{(N-\Delta) \operatorname{Var}(\hat{h})}, \\
\operatorname{Var}(\hat{h}) & =\frac{1}{N} \sum_{i=1}^{N}\left(\hat{h}_{i}-\bar{h}\right)^{2}, \quad \bar{h}=\frac{1}{N} \sum_{i=1}^{N} \hat{h}_{i} .
\end{aligned}
$$

If $\hat{h}_{i} \equiv h\left(t_{i}\right)$, for a periodic solution $h$ with period $P=\delta \Delta$, we have $R_{\Delta}=0$. In numerical experiments, we can only guarantee that the $\Delta$-discrepancy of a periodic solution is small enough: $R_{\Delta}<r_{\epsilon}=4 \epsilon^{2} / \operatorname{Var}(\hat{h})$, where $\epsilon$ is the absolute numerical precision. We call near-period the minimal number $\mathcal{P}=\delta \Delta$ such that

$R_{\Delta}<r_{\eta}$ and $R_{\Delta}<R_{\Delta \pm 1}$

for some prescribed $0<\eta \ll 1$. The first condition ensures that the $\Delta$-discrepancy $R_{\Delta}$ is small enough, while the second one guarantees that $R_{\Delta}$ is a local minimum as a function of $\Delta$.

The following proposition follows readily from the definition of $\mathcal{P}$.

Proposition 2 (Convergence theorem). The near-period $\mathcal{P}$ converges to the actual period $T$ of a continuous periodic function $h(t)$ when the numerical step $\delta$ and nominal accuracy $\epsilon$ decrease: $\lim _{\delta \rightarrow 0, \epsilon \rightarrow 0} \mathcal{P}=T$.

The continuity requirement in the hypothesis is too restrictive for this statement, but it suffices for our purpose, since solutions of model Eq. (6), for a given triplet $(b, \tau, \kappa)$, are smooth. The rate of convergence in Proposition 2 depends strongly on the period structure of $h(t)$. The rate is high for functions with "simple" periods, e.g. with a single local maximum within the period $T$, and may be arbitrarily low in the general case; e.g. for $h=h_{1}+\gamma h_{2}$, where $h_{1}$ has period $T / 2$, $h_{2}$ period $T$, and $\gamma$ is small enough, the convergence is quite slow.

To summarize, the near-period $\mathcal{P}$ approximates the actual period $T$ for periodic functions. The functional $\mathcal{P}$ can also be defined for certain functions that are not periodic. For instance, this may be the case for a quasiperiodic function $h(t)=p_{1}(t)+p_{2}(t)$, where each $p_{i}(t)$ is periodic and the two periods, $T_{1}$ and $T_{2}$, are rationally independent. It is also the case for a near-periodic function $h(t)=p_{0}(t)+p_{1}(t)$, where $p_{0}(t)$ is periodic, and $p_{1}(t)$ has sufficiently small amplitude. As we shall see, the period approximation $\mathcal{P}$ is quite helpful in understanding the structure of our model's solution set.

\subsection{Small forcing amplitude and frequency locking}

We mentioned in Sect. 2.2 that, without external forcing $(b=0)$, the nontrivial stable solutions of the model Eqs. (6)(7) are periodic with period $4 \tau$. When the external forcing

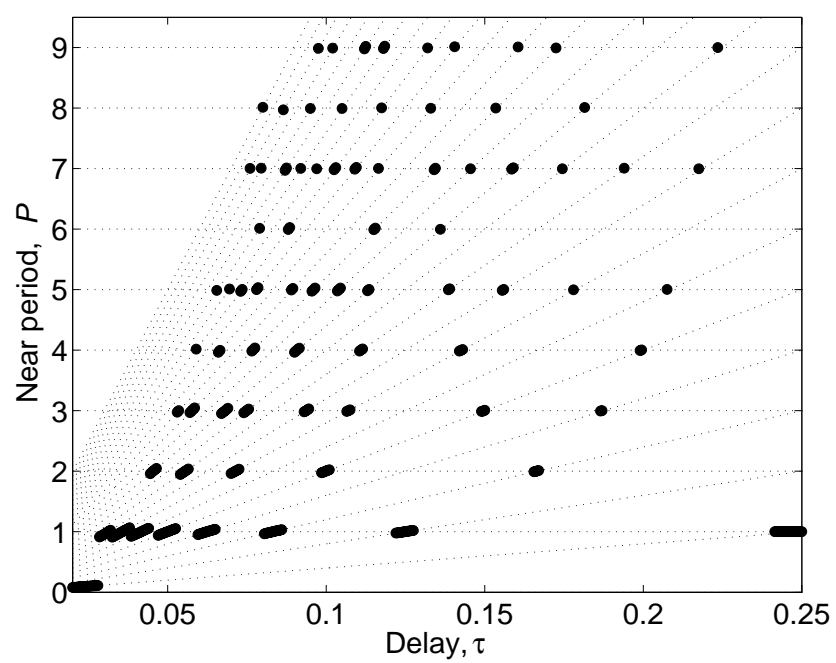

Fig. 3. Near-period $\mathcal{P}$ as a function of delay $\tau$ for fixed $b=0.03$, heavy solid segments; dashed straight lines correspond to $\mathcal{P}=4 \tau k$, $k=1,2, \ldots$. The near-period is always a multiple of $4 \tau$ close to an integer.

is small, $b \ll 1$, the dynamical system tries to retain this property. Figure 3 shows the near-period $\mathcal{P}$ as a function of the delay $\tau$ for fixed $b=0.03$ and $\kappa=100$. Here $r_{\eta}=R_{1}$, that is we compare the $\Delta$-discrepancy $R_{\Delta}$ with the one-step discrepancy $R_{1}$; the latter measures the degree of continuity of our discrete-time approximation to $h(t)$. Straight dashed lines in the figure correspond to $\mathcal{P}=4 \tau k$ for positive integer $k$. One can see that the solution's near-period is always a multiple of $4 \tau$ and always close to an integer. This state of affairs is a natural compromise between the internal period $4 \tau$ and the driving period 1 , a compromise rendered possible by the internal oscillator's nonlinearity.

More generally, Fig. 4 shows the map of the period in$\operatorname{dex} k=\mathcal{P} /(4 \tau)$ in the $b-\tau$ plane for $0<b<0.03,0<\tau<0.22$. Here one immediately recognizes the so-called Devil's bleachers scenario of transition to chaos, documented in other ENSO models, including both ICMs (Jin et al., 1994, 1996; Tziperman et al., 1994, 1995; Ghil and Robertson, 2000) and GCMs (Ghil and Robertson, 2000), as well as in certain observations (Ghil and Robertson, 2000; Yanai and $\mathrm{Li}, 1994)$. The periodically forced model Eq. (6) exhibits the web of resonances that characterizes coupled oscillators, although the "external oscillator" quickly wins over the internal one as the amplitude $b$ of the forcing increases. For $b=0.1$ (not shown) the near-period plot looks similar to the one shown in Fig. 3, but $\mathcal{P}$ takes only integer values. When the forcing amplitude increases further, the near-period $\mathcal{P}$, as well as the actual period $T$, is locked solely to integer values.

\subsection{Onset of the instabilities}

Tziperman et al. (1994) reported that the onset of chaotic behavior in their two-delay, periodically forced DDE model is 


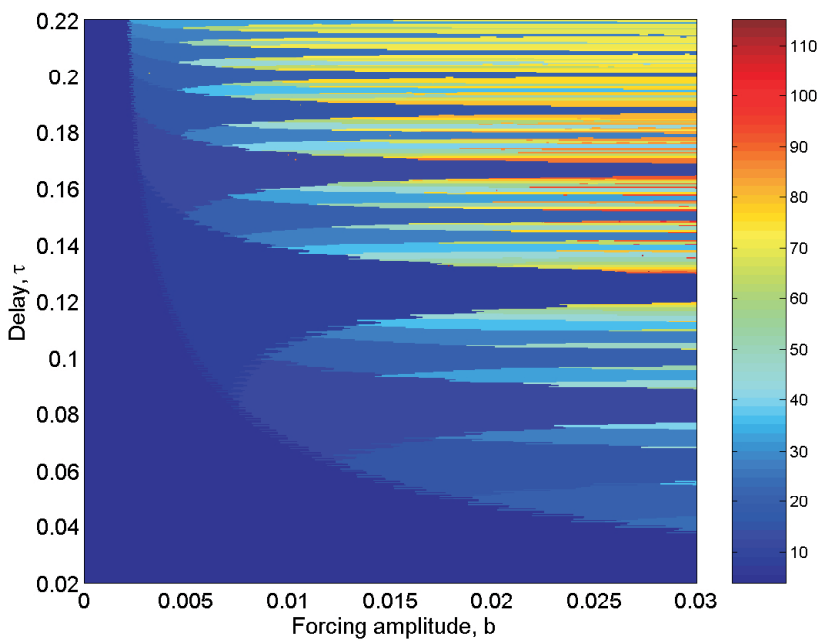

Fig. 4. Devil's bleachers: period index $k=\mathcal{P} /(4 \tau)$ as a function of forcing amplitude $b$ and delay $\tau$. Notice the presence of very long periods, of 100 years and more, in the color bar and figure.

associated with the increase of the atmosphere-ocean coupling $\kappa$; Munnich et al. (1991) made a similar observation for an iterated-map model of ENSO. We explore this transition to chaos in our model over its entire, 3-D parameter space.

First, we compute in Fig. 5 the trajectory maximum $M$ as a function of the parameters $b$ and $\tau$ for increasing values of $\kappa$. For small values of coupling (top panel) we have a smooth map, monotonously increasing in $b$ and periodic with period 1 in $\tau$. As the coupling increases, the map loses its monotonicity in $b$ and periodicity in $\tau$ for large values of $\tau$, but it is still smooth. For $\kappa \approx 2$ (middle panel), a neutral curve $f(b, \tau)=0$ emerges that separates a smooth region (to the right of the curve), where we still observe monotonicity in $b$ and periodicity in $\tau$, from a region with rough behavior of $M$. The gradient of $M(b, \tau)$ is quite sharp across this neutral curve.

Further increase of the coupling results in a qualitative change in the maximum map. The neutral curve, which becomes sharp and rough, separates two regions with very different behavior of $M(b, \tau)$ (bottom panel). To the right of the curve, the map $M(b, \tau)$ is still smooth, periodic in $\tau$ and monotonic in $b$. To the left, one sees discontinuities that produce rough and complicated patterns. The mean position of the neutral curve $f(b, \tau)=0$ quickly converges to a fixed profile, although its detailed shape at smaller scales continues to change with increasing $\kappa$. The limiting profile is close to the one observed for $\kappa=11$ (bottom panel).

\subsection{Unstable behavior}

In this subsection we illustrate the model's parametric instabilities using the four trajectory statistics introduced in Sect. 3.1: maximum $M$, mean $E$, mean of positive values
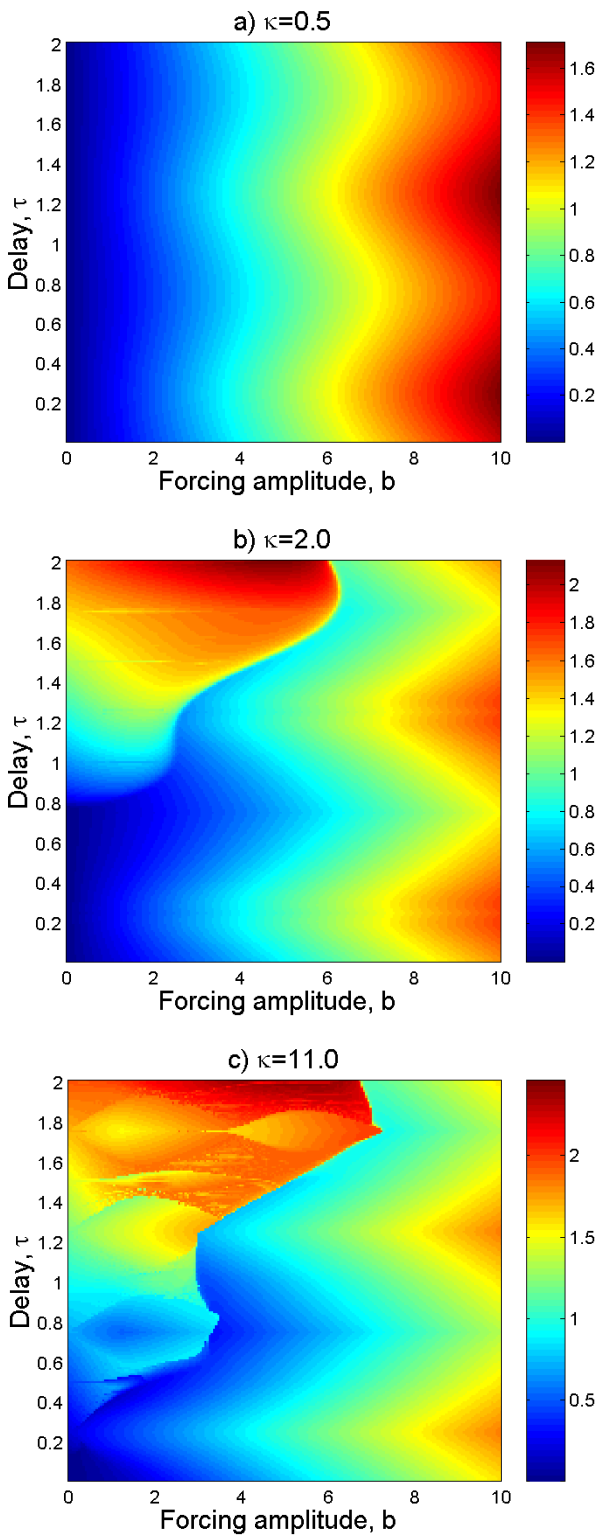

Fig. 5. Maximum map $M=M(b, \tau)$. Top: $\kappa=0.5$, middle: $\kappa=2$, and bottom: $\kappa=11$. Notice the onset of instabilities and emergence of a neutral curve $f(b, \tau)=0$ that separates the smooth from the unstable regions.

$E_{+}$, and near-period $\mathcal{P}$. Figure 6 shows a plot of these statistics in a rectangle of the plane $(b, \tau)$ for fixed $\kappa=10$. The neutral curve of Fig. 5c crosses this rectangle from its bottom left corner to the central point on its right edge; thus the bottom right region of each panel corresponds to smooth behavior of each statistic map, while the top left region corresponds to rough behavior. This figure illustrates the following points:

The maximum map $M(b, \tau)$ (Fig. 6a) shows, among other instabilities, a pronounced jump along a mainly horizontal curve in the $(b, \tau)$ plane. In the vicinity of this curve, 

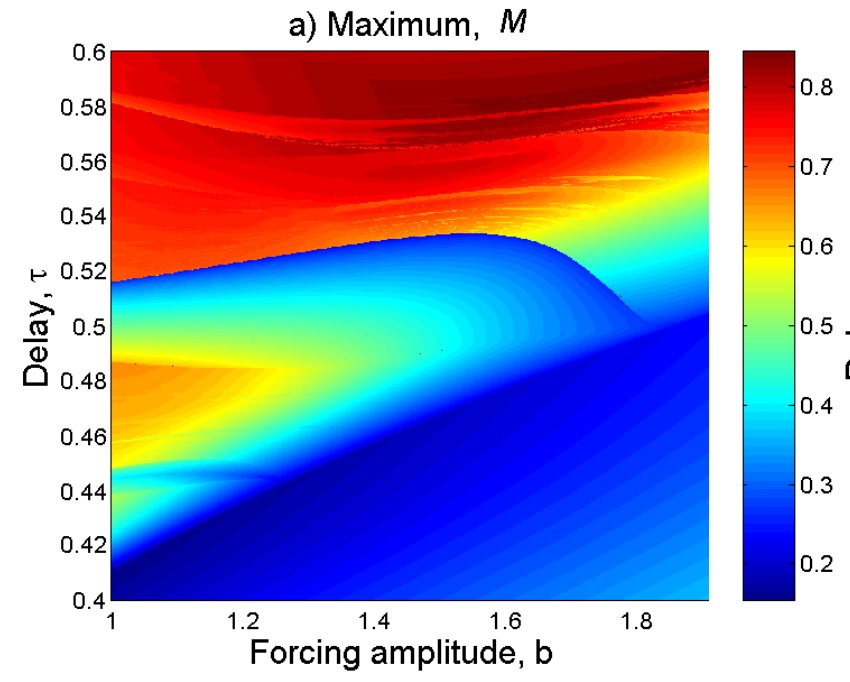

c) Mean of positive values, $E_{+}$
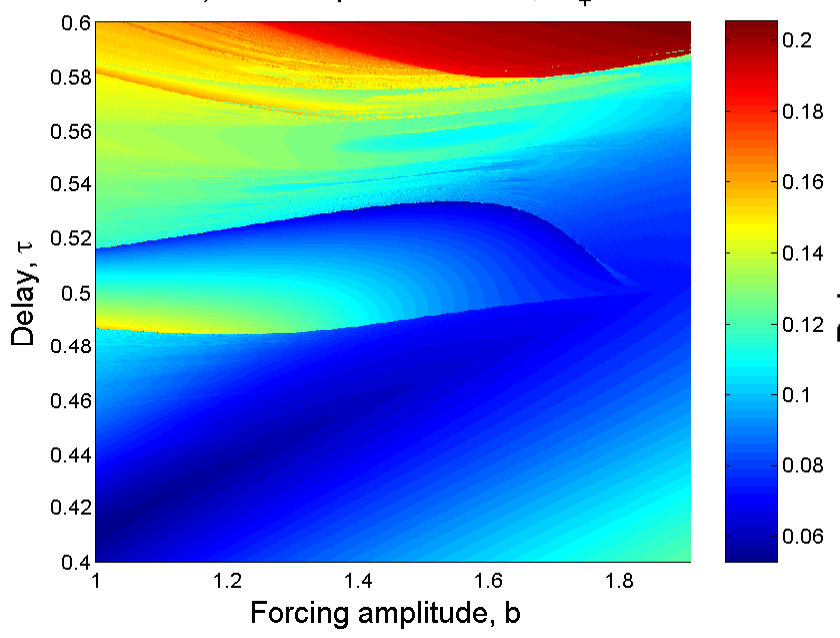

b) Mean, $E$

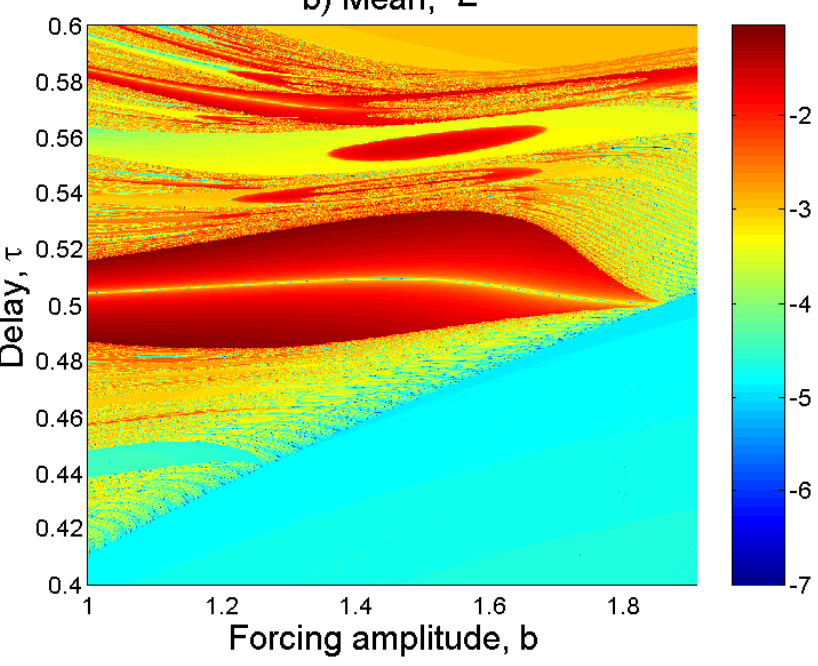

d) Near-period, $P$

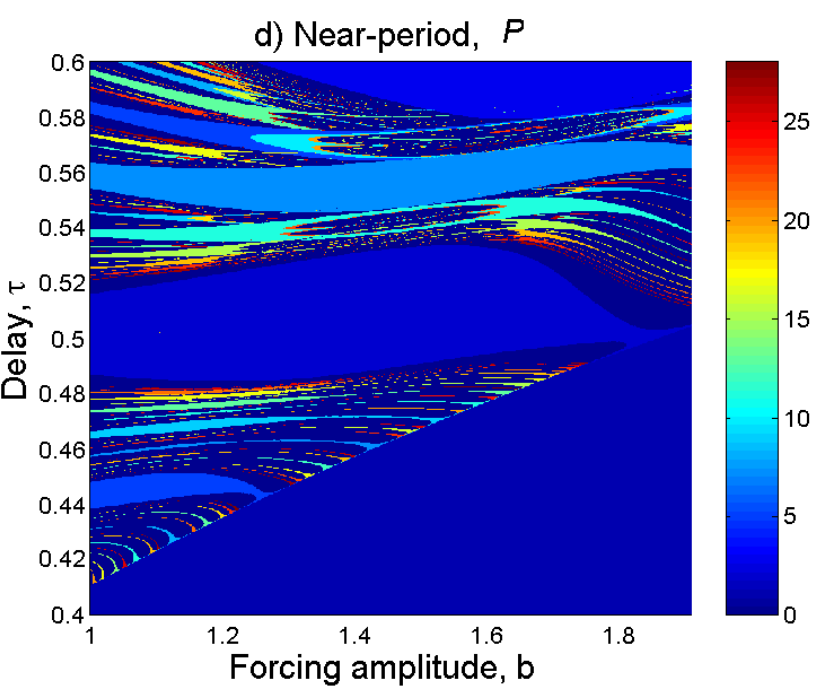

Fig. 6. Trajectory statistics plots for $\kappa=10$, as a function of forcing amplitude $b$ and delay $\tau$ : (a) maximum map, $M(b, \tau)$; (b) mean map, $\log |E(b, \tau)| ;(\mathbf{c})$ mean of positive values, $E_{+}(b, \tau)$; and $(\mathbf{d})$ near-period map, $\mathcal{P}(b, \tau)$.

an arbitrarily small increase in $\tau$ causes a $200-300 \%$ jump (from 0.25 to $0.5-0.8$ ) in $M$. A pair of trajectories on either side of this transition is shown below in Fig. 9.

The mean map $E(b, \tau)$ (Fig. 6b). To emphasize the parametric instabilities, we show here $\log _{10}|E|$. Deviations of $E$ from 0 reflect the trajectory's asymmetry; hence the larger values of this map indicate asymmetric solutions. In this experiment, we use a numerical precision of $10^{-4}$, so that values of $\log _{10}|E|<-4$ effectively correspond to symmetric trajectories, $E=0$. One can see that the symmetry, characteristic for trajectories from the smooth region (bottom right part), breaks across the neutral curve. In the unstable region, the magnitude of the asymmetry is very intermittent; it ranges over three orders of magnitude, taking its maximal value in the region that corresponds to the jump in the trajectory maximum, cf. panel (a).
The mean of positive values $E_{+}(b, \tau)$ (Fig. 6c), by comparison with the maximum map in panel (a), shows that certain internal instabilities may affect trajectory shape without affecting the behavior of extremes. For instance, the maximum map $M(b, \tau)$ is smooth within the neighborhood of the point $(b=1.5, \tau=0.49)$, although the map $E_{+}(b, \tau)$ exhibits a discontinuity across this neighborhood. In fact, one arrives at the same conclusion by comparing the maximum map to the mean map, cf. panels (a) and (b), respectively.

The near-period map $\mathcal{P}(b, \tau)$ (Fig. 6d). The near-period is varying over the interval $[0,27]$ in this map. As we have noticed, not all of these values correspond to trajectories that are actually periodic, rather than just nearly so (see Sect. 3.1) - The large constant regions, though, do reflect the actual periods; as a rule, they correspond to small values of $\mathcal{P}$. Examples include: $\mathcal{P}=1$, within the smooth part of the map 

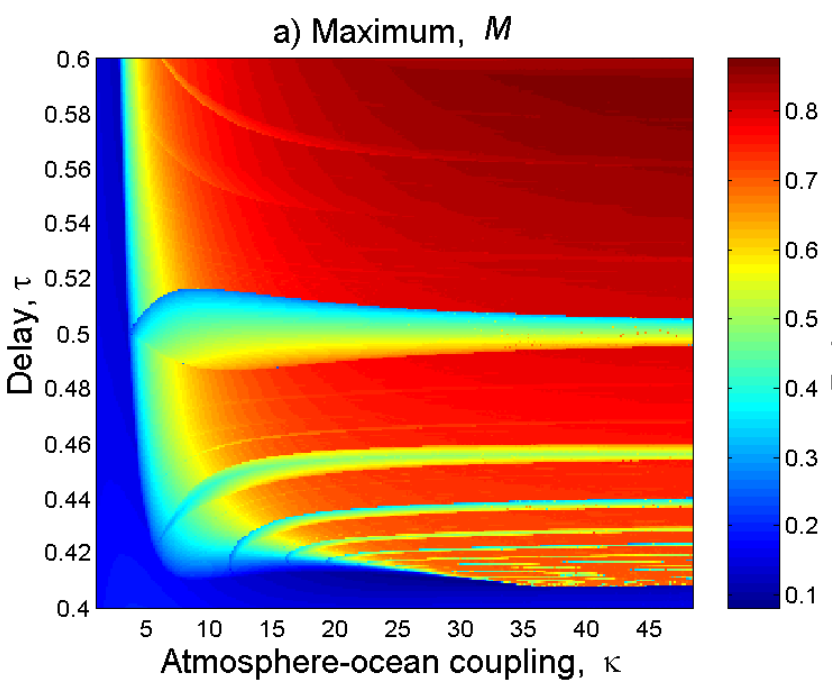

c) Mean of positive values, $E_{+}$

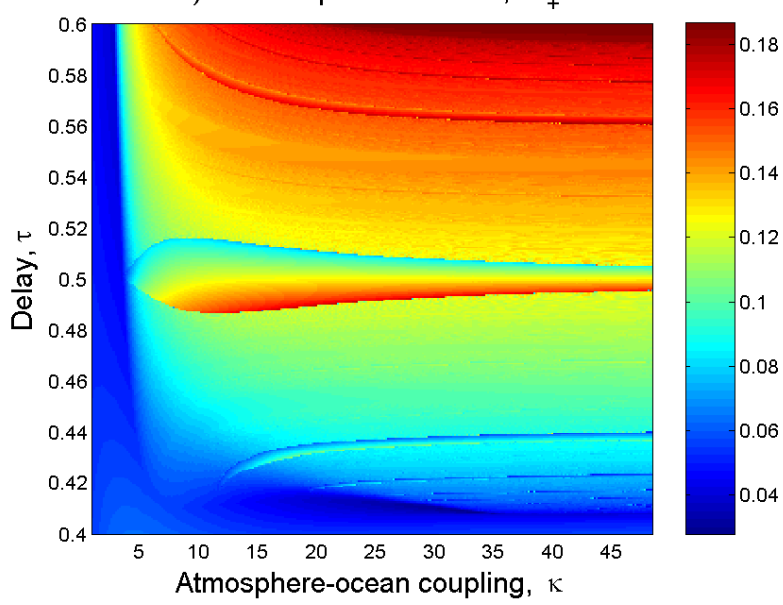

b) Mean, $E$

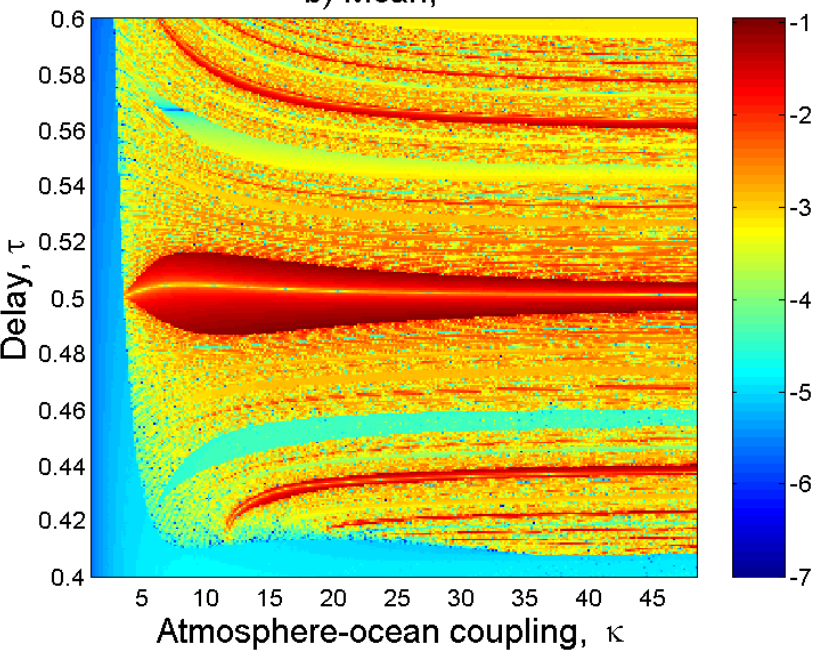

d) Near-period, $P$

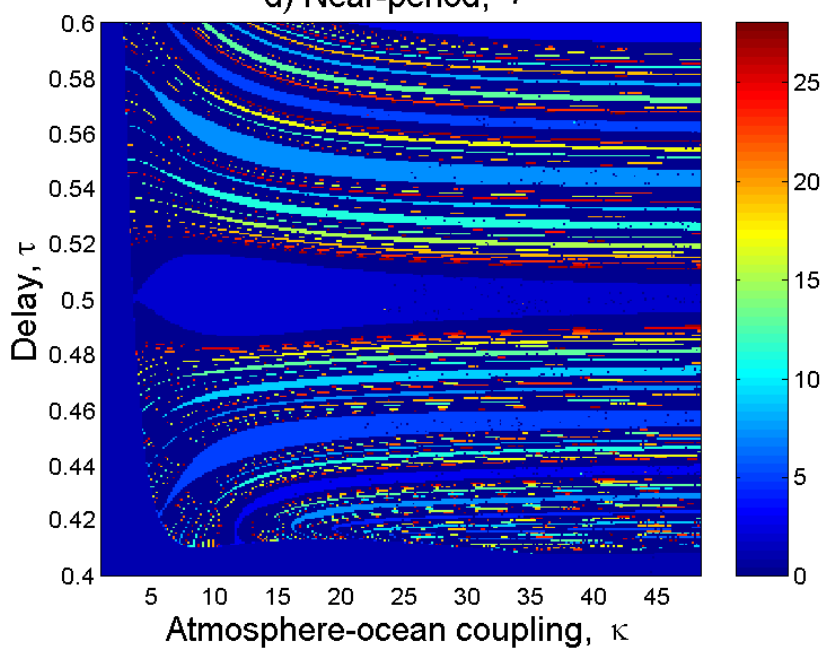

Fig. 7. Solution statistics plots for $b=1$, as a function of coupling parameter $\kappa$ and delay $\tau$. Same panels as in Fig. 6 .

(bottom right); $\mathcal{P}=2$ within the middle horizontal tongue; $\mathcal{P}=3$ within the top right part; and $\mathcal{P}=5$ in a small tongue that touches the left margin of the plot at $(b=1, \tau=0.44)$.

Figure 7 shows the same plots of solution statistics over a rectangle of the plane $(\kappa, \tau)$, for a fixed value $b=1$; it illustrates the onset and development of instabilities as a function of the coupling parameter $\kappa$. Comments similar to those above, which concern the behavior of individual maps and the connections between them, apply to this set of maps as well.

Figure 8 shows the maximum and mean maps for $\kappa=100$. The increase in the atmosphere-ocean coupling, with its associated nonlinearity, amplifies the model's instabilities and leads to more complex dynamics that is quite chaotic. The rigorous verification of the chaotic properties is left, however, for future work.
Figure 9 shows three examples of change in solution behavior across the neutral curve that separates smooth from rougher behavior. In all three cases shown, one trajectory (dashed line) has period one and lies within the smooth part of the parameter space, in the immediate vicinity of the neutral curve, while the other trajectory (solid line) corresponds to a point in parameter space that is quite close to the first one but on the other side of the neutral curve.

Panel (a) illustrates transition to quasiperiodic behavior with a "carrier wave" of period near 8 , alternating smoothly 3 warm and 3 cold events, separated by one "normal" year. In panel (b) we see single large El Niños alternating with single large La Niñas, separated by 3 normal years. Panel (c) exhibits interdecadal variability, like in Fig. 2e, except that the transitions between warm and cold "decades" is sharper here, and the spells of El Niños and La Niñas even longer (a dozen years here vs. roughly ten there). 
a) Maximum, $M$

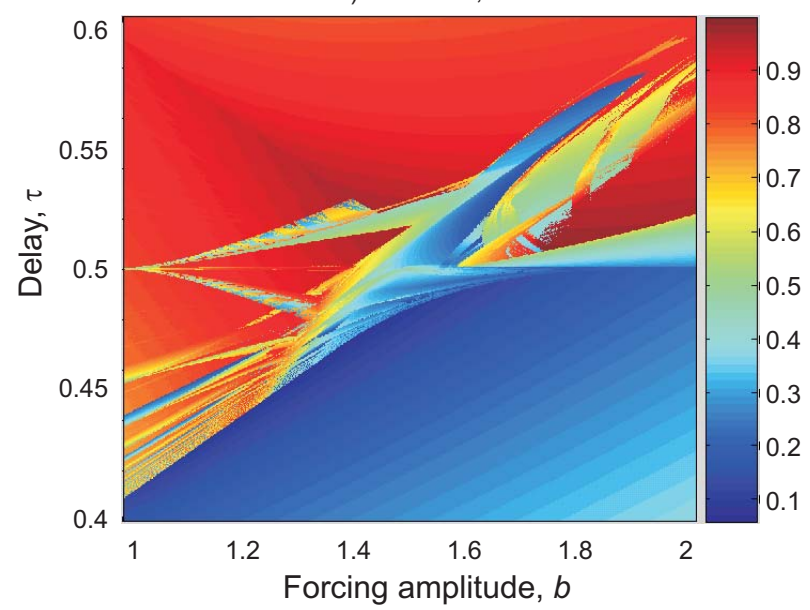

b) Mean, $E$

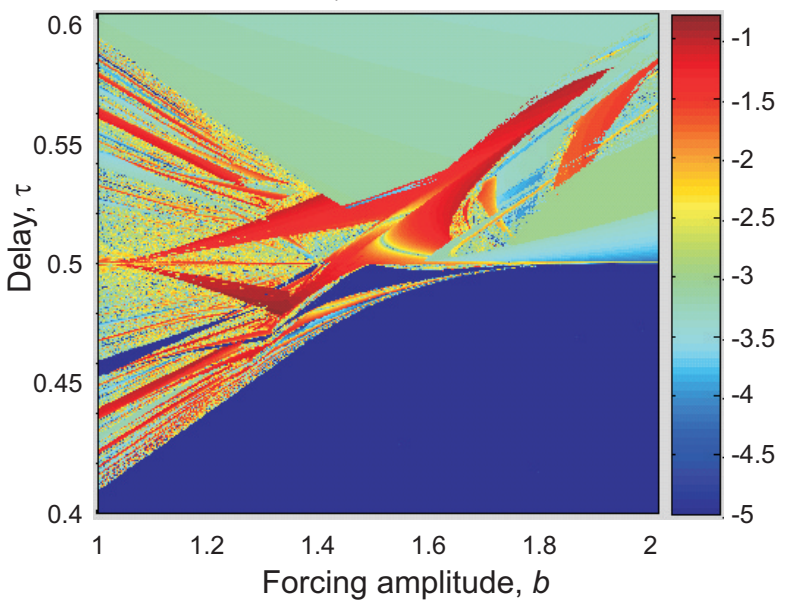

Fig. 8. Solution statistics plots for $\kappa=100$ : (a) maximum map, $M(b, \tau)$; and (b) mean map, $\log |E(b, \tau)|$.

\section{Discussion}

We have considered a toy model for ENSO variability that is governed by a delay differential equation (DDE) with a single, fixed delay and additive periodic forcing. Thus, we follow a line of research pioneered by Suarez and Schopf (1988), Battisti and Hirst (1989), and Tziperman et al. (1994), who have shown that DDE models can effectively capture complex phenomena found in much more detailed ENSO models, as well as in observational data sets. DDE models are very simple and, at the same time, exhibit rich and complex behavior. Stability and bifurcation analysis for such models can be carried out analytically only to some extent, but numerical methods are being actively developed (Baker, 2000; Baker et al., 1995; Engelborghs et al., 2001), and we have not yet taken full advantage here of either approach.

Our toy model necessarily ignores a multitude of actual physical mechanisms and processes that might affect ENSO dynamics. For example, an alternative to the additive forcing, currently used in Eq. (4), could be a seasonal modulation of the air-sea coupling coefficient $\kappa$, which seems to be better justified by the physics of the problem. It would be equally interesting to see how the inclusion of a positive feedback, associated with the Kelvin wave, might affect the model dynamics. These and other modifications of the current basic Eq. (4) seem to deserve careful exploration.

To initiate stability and bifurcation analysis of ENSOrelated DDE models, we started here with a descriptive numerical exploration of Eqs. (6)-(7) over a wide range of physically relevant parameter values. We studied parameter dependence of various trajectory statistics, and report the existence of a large domain in parameter space where the statistics maps are strikingly discontinuous. The local continuousdependence theorem (Proposition 1) suggests, at least, that the reported discontinuities in global solutions point to the existence of unstable solutions of Eqs. (6)-(7); the complex discontinuity patterns (see Figs. 6 and 7) lead us to suspect the presence of a rich family of unstable solutions that underlie a complicated attractor. It would be interesting to study in greater detail the unstable solutions and understand their role in shaping the system dynamics.

Summarizing the model results in terms of their relevance for ENSO dynamics, we emphasize the following observations. A simple DDE model Eqs. (6)-(7) with a single delay reproduces the Devil's staircase scenario documented in other ENSO models, including ICMs and GCMs, as well as in observations (Jin et al., 1994, 1996; Tziperman et al., 1994, 1995; Ghil and Robertson, 2000). The model illustrates, in simplest possible terms, the role of the distinct parameters: strength of seasonal forcing $b$ vs. oceanatmosphere coupling $\kappa$ and transit time $\tau$ of oceanic waves across the Tropical Pacific.

We find spontaneous transitions in mean thermocline depth, and hence in sea surface temperature (SST), as well as in extreme annual values; these transitions occur for purely periodic, seasonal forcing. The model generates intraseasonal oscillations of various periods and amplitudes, as well as interdecadal variability. The latter result suggests that interdecadal variability in the extratropical, thermohaline circulation (Dijkstra, 2005; Dijkstra and Ghil, 2005) might interfere constructively with ENSO's intrinsic variability on this time scale.

The former result might likewise suggest that MaddenJulian oscillations (Madden and Julian, 1971, 1972, 1994) and westerly wind bursts (Gebbie et al., 2007; Harrison and Giese, 1988; Verbickas, 1998; Delcroix et al., 1993) in the Tropical Pacific are affected by ENSO's interannual modes at least as much as they affect them in turn. Boulanger et al. (2004) and Lengaigne et al. (2004), among others, provide a comprehensive discussion of how weather noise could 


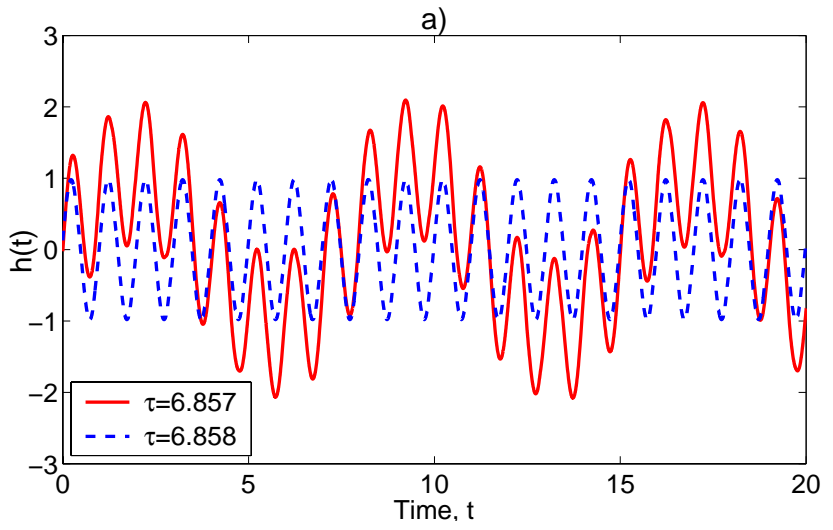

b)

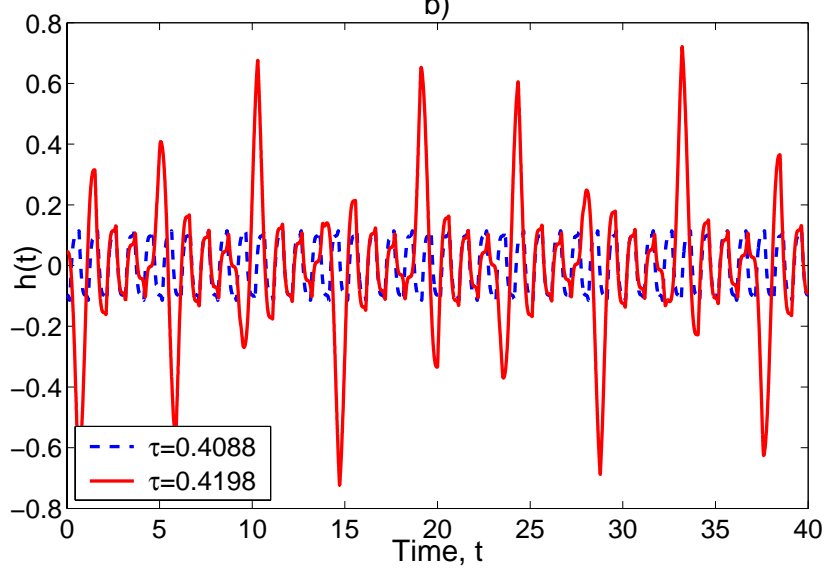

c)

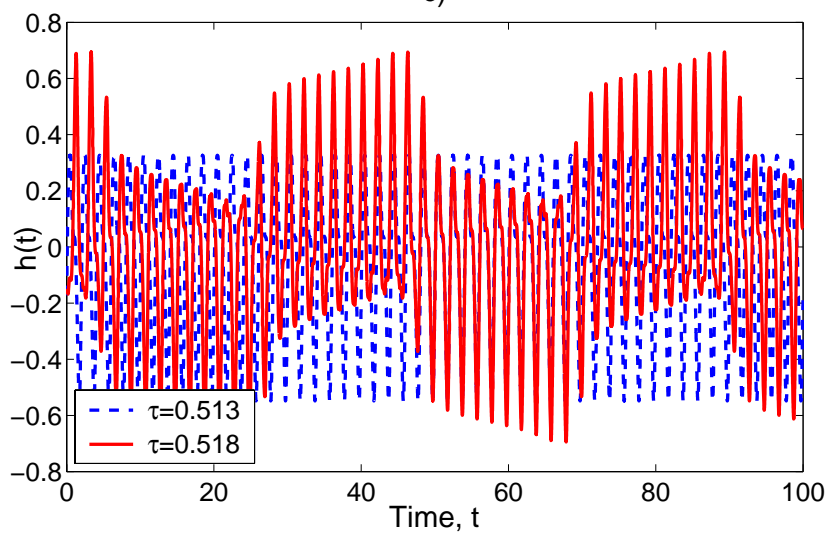

Fig. 9. Examples of instabilities across the neutral curve that separates smooth from rough behavior; see Fig. 5. Panels (a) and (b) $b=1, \kappa=100$; panel (c) $b=1, \kappa=10$. The dashed blue curve shows a period-1 trajectory on the smooth side of the neutral curve; the solid red curve shows a trajectory immediately across the neutral curve, in the rough-solution domain.

be responsible for the complex dynamics of ENSO, and, in particular, whether wind bursts could trigger El Niño events. Arguments about a "stochastic paradigm" for ENSO, with linear or only mildly nonlinear dynamics being affected decisively by weather noise, vs. a "deterministically chaotic paradigm," with decisively nonlinear dynamics, have also been discussed by Ghil and Robertson (2000).
A sharp neutral curve in the $(b-\tau)$ plane separates smooth parameter dependence in the solutions' map of "metrics" (Taylor, 2001; Fuglestvedt et al., 2003) from "rough" behavior. We expect such separation between regions of smooth and rough dependence of solution metrics on parameters in much more detailed and realistic models, where it is harder to describe its causes as completely.

Finally, it appears that, even in as simple a model as our DDE, the mean, extrema and periodicity of solutions can change (a) spontaneously, without any change in the external forcing; and (b) one of these characteristics can change considerably, while others change but very little. Furthermore, certain parts of parameter space involve only small and smooth changes, while others involve large and sudden ones. It is quite conceivable that such behavior might arise in intermediate climate models (Jin et al., 1996; Neelin et al., 1994, 1998) and GCMs (Murphy et al., 2004; Stainforth et al., 2005).

\section{Appendix A}

\section{A simple example of DDE complexity}

In his classical book (Hale, 1977) on functional differential equations, Jack Hale remarks that systematic study of differential equations with dependence on the past started with the work of Volterra on predator-prey models and viscoelasticity at the beginning of the 20th century. DDEs have thus been actively studied and applied for almost a century. Still, they are a relatively new modeling tool when compared to ODEs, and their theory and numerical analysis are much less developed than for ODEs. To develop the reader's intuition for DDEs, we discuss in this appendix a simple autonomous ODE and the corresponding DDE obtained by introducing a fixed time delay; our goal is to illustrate how this apparently innocuous modification complicates the solution set of the equation and renders its analytical and numerical study more elaborate.

We start with the linear, scalar ODE

$\dot{x}(t)=\alpha x(t)$

Assuming a solution of the form $x=c e^{\lambda t}$, we substitute it in Eq. (A1) to find its characteristic equation

$\lambda=\alpha$.

This equation allows us to find all possible functions of the given form that satisfy Eq. (A1). Clearly, fixing an initial condition $x(0)=x_{0}$ leaves only one solution with $c=x_{0}$. This example illustrates an important general property of autonomous ODEs: their characteristic equations are polynomials in $\lambda$ and thus have a finite set of (complex-valued) solutions that can be easily found. As a result, the finite set of solutions to Eq. (A1) can also be described easily. 
Let us introduce now a delay $\tau$ into Eq. (A1):

$\dot{x}(t)=\alpha x(t-\tau)$.

This modification implies that it takes some finite time $\tau$ for changes in the model state $x(t)$ to affect its rate of change $\dot{x}(t)$. Such an assumption makes sense in many applications, with numerous specific examples given in the Introduction to Hale (1977), and in Kolmanovskii and Nosov (1986). Proceeding as before, we assume a solution of the form $x=c e^{\lambda t}$ and obtain the characteristic equation

$\lambda e^{\lambda \tau}=\alpha$

The fact that not all exponential terms cancel out in Eq. (A3) changes dramatically the solution set of this characteristic equation. It can be shown that Eq. (A3) has an infinite number of complex solutions; hence there exists an infinite number of functions that satisfy Eq. (A2) (Hale, 1977; Falbo, 1995). A general solution to Eq. (A2) is given by (Falbo, 1995):

$$
\begin{aligned}
x(t) & =C_{0} e^{-t / \tau}+C_{1} e^{r_{0} t}+C_{2} e^{r_{1} t}+C_{3} e^{r t} \\
& +\sum_{k=1}^{\infty} e^{p_{k} t}\left[C_{1 k} \cos \left(q_{k} t\right)+C_{2 k} \sin \left(q_{k} t\right)\right] .
\end{aligned}
$$

Here $p_{k} \pm i q_{k}$ are complex solutions of Eq. (A3), $C_{1 k}$ and $C_{2 k}$ are arbitrary constants, and $C_{0}, C_{1}, C_{2}, C_{3}$ depend on the values of $\alpha$ and $\tau$ : for $\alpha<-1 /(\tau e), C_{i}=0, i=0,1,2,3$; for $\alpha=-1 /(\tau e), C_{0}$ is arbitrary, and $C_{i}=0, i=1,2,3$; for $-1 /(\tau e)<\alpha<0, C_{0}=C_{3}=0, C_{1}, C_{2}$ are arbitrary, and $r_{0}$, $r_{1}$ are the real roots of Eq. (A3); finally for $\alpha>0, C_{i}=0$ for $i=0,1,2, C_{3}$ is arbitrary, and $r$ is the only real root of Eq. (A3). Accordingly, this simple autonomous linear DDE may have increasing, decreasing, and oscillating solutions.

The "burst" of complexity in the solution set of Eq. (A2) compared to that of Eq. (A1) is pervasive in the DDE realm. Solving characteristic equations arising from DDEs is typically quite involved and requires usually some general results on equations that mix polynomial and exponential terms (see, e.g., Appendix A in Hale, 1977). Stability and bifurcation studies for DDEs face a similar "burst" of complexity, compared to ODEs.

Numerical exploration of DDEs is also considerably more complicated than for ODEs. The question of discontinuities in the solutions' derivatives is crucial: Because general numerical methods for both ODEs and DDEs are intended for problems with solutions that have several continuous derivatives, discontinuities in low-order derivatives (up to the order of the integration method) require special attention. Such discontinuities are not all that rare for ODEs, but they are almost always present for DDEs, since the first derivative of the history function at the initial point is almost always different from the first derivative of the solution there. Moreover, discontinuities are a much more serious matter for DDEs than they are for ODEs because they propagate: the smoothness of the derivative $\dot{x}$ at the current time $t$ depends on the smoothness of the solution $x$ at past times in the interval $t-\tau$.

This difficulty may be illustrated using our very simple example

$\dot{x}(t)=x(t-1)$,

where we set, without loss of generality, $\tau=\alpha=1$. For this equation $x^{(k+1)}(t)=x^{(k)}(t-1)$, where $x^{(k)}$ denotes the $k$-th derivative. In general, if there is a discontinuity of order $k$ at a time $t^{*}$, meaning that $x^{(k)}$ has a jump at $t=t^{*}$, then as $t$ crosses the value $t^{*}+1$, there is a discontinuity in $x^{(k+1)}$ because of the term $x(t-1)$. With multiple delays $\tau_{i}$, a discontinuity at time $t^{*}$ propagates to the times

$t^{*}+\tau_{1}, t^{*}+\tau_{2}, \ldots, t^{*}+\tau_{k}$,

and each of these discontinuities in turn propagates. If there is a discontinuity of order $k$ at time $t^{*}$, the discontinuity at each of the times $t^{*}+\tau_{j}$ is at least of order $k+1$. Because the effect of a delay appears in a higher-order derivative, the solution does become smoother as the integration proceeds. This "generalized smoothing" proves to be quite important to the numerical solution of DDEs.

To illustrate these statements, suppose we wish to solve Eq. (A5) with history $x(t) \equiv 1$ for $t \leq 0$. On the interval $0 \leq t \leq 1$ the solution is $x(t-1)=1$ because $t-1 \leq 0$. Thus, the DDE on this interval reduces to the ODE $\dot{x}(t)=1$ with initial value $x(0)=1$. We solve this problem to obtain $x(t)=t+1$ for $0 \leq t \leq 1$. Notice that this solution exhibits a typical discontinuity in its first derivative at $t=0$ because it is zero to the left of the origin and unity to the right. Now that we know the solution for $t \leq 1$, we can reduce the DDE on the interval $1 \leq t \leq 2$ to an ODE $\dot{x}=(t-1)+1=t$ with initial value $x(1)=2$ and solve this problem to find that $x(t)=0.5 t^{2}+1.5$ on this interval. The first derivative is continuous at $t=1$, but there is a discontinuity in the second derivative. In general, the solution of the DDE on the interval $[k, k+1]$ is a polynomial of degree $k+1$ and the solution has a discontinuity of order $k+1$ at time $t=k$.

In order for a solver to account for these specific features of DDEs and to solve them efficiently, accurately and reliably, there must be a great deal of care taken "under the hood" of the solver. Discontinuities need be tracked only up to the order of the integration method, since higher-order discontinuities do not affect the performance of the solver, i.e. its procedures for error estimation and step size control. Our solver of choice, dde_solver, tracks discontinuities explicitly and includes them as integration grid points, in order to avoid interpolating across them. For problems with constant delays, it is possible to build the necessary "discontinuity tree" in advance and to step exactly to each point in the tree.

For problems with state-dependent delays, the discontinuity times are not known in advance. In this case, dde_solver tracks discontinuities using root finding, in conjunction with 
the primary integration method's underlying polynomial interpolants, to locate the discontinuities and restart the integration at each such point. Some other available solvers handle discontinuity propagation differently; but the best solvers do take special precautions of one kind or another, since ignoring discontinuities can significantly affect the reliability of a DDE solver. We refer in this context also to several distinct approaches to the numerical solution of Boolean delay equations (Dee and Ghil, 1984; Ghil and Mullhaupt, 1985; Saunders and Ghil, 2001; Zaliapin et al., 2003).

\section{Appendix B}

\section{Proof of Proposition 1}

Consider the IVP Eq. (6)-Eqs. (7), with the rhs of the DDE Eq. (6) denoted by

$\mathcal{F}(t, h(\cdot))=-\tanh [\kappa h(\cdot)]+b \cos (2 \pi t)$.

Existence of the solution to this problem on $[0, T]$ for some $T>0$ readily follows from the continuity of $\mathcal{F}(t, h)$ and the general existence theorem for DDEs (Hale (1977), Theorem 2.1, p. 41). Moreover, Nussbaum (Nussbaum, 1998, Theorem 1, p. 3) remarks that if there exist constants $A$ and $B$ such that $\|\mathcal{F}(t, h)\| \leq A\|h\|+B$ for all $(t, h) \in \mathbb{R} \times X$ then one can choose $T=\infty$. Since $|\tanh (\kappa z)| \leq \kappa|z|$, the choice of $A=\kappa$ and $B=b$ ensures the solution's existence on $[0, \infty)$.

Uniqueness could be derived from the Lipschitz property of $\mathcal{F}(t, h)$ in $h$ and the general uniqueness theorem (Hale, 1977, Theorem 2.3, p. 42). However, for our system the uniqueness can be established in a simpler way. Indeed, assume that $x(t)$ and $y(t)$ are solutions of Eqs. (6)-(7) on $[0, T]$, with rhs $\mathcal{F}(t, h(t-\tau))$ and the initial condition $\phi(t)$. Then, for $0<t \leq \tau$,

$$
\begin{aligned}
& x(t)-y(t)=\int_{0}^{t}[\mathcal{F}(s, x(s-\tau)-\mathcal{F}(s, y(s-\tau)] d s \\
& =\int_{0}^{t}(\tanh [\kappa y(s-\tau)]-\tanh [\kappa x(s-\tau)]) d s \\
& =\int_{-\tau}^{t-\tau}(\tanh [\kappa y(u)]-\tanh [\kappa x(u)]) d u \equiv 0 .
\end{aligned}
$$

Thus, the solutions $x$ and $y$ are identical up to $t=\tau>0$. The uniqueness is proven by successively advancing in time by intervals of length $\tau$.

Continuous dependence on initial conditions and the rhs of Eq. (6) for any finite $T$ follows from the existence and uniqueness and the general continuous dependence theorem (Hale, 1977, Theorem 2.2, p. 41). To show the continuous dependence on the delay $\tau$, also for finite $T$, we consider the sequence $\tau_{k} \rightarrow \tau, k \rightarrow \infty$, and for any $\tau_{k}$ introduce the time scale change: $s_{k}=t \tau / \tau_{k}$.
Then,

$h\left(t-\tau_{k}\right)=h\left(\frac{\tau_{k}}{\tau}\left(s_{k}-\tau\right)\right) \equiv h_{k}\left(s_{k}-\tau\right)$,

and one finds

$\frac{d}{d s_{k}} h_{k}\left(s_{k}\right)=-\frac{\tau_{k}}{\tau} A_{\kappa}\left[h_{k}\left(s_{k}-\tau\right)\right]+b \cos \left(2 \pi \frac{s_{k} \tau_{k}}{\tau}\right)$.

Clearly, the rhs of this system converges to $\mathcal{F}$ as $k \rightarrow \infty$. This shows that a small change in the delay $\tau$ can be considered as a small change of the rhs of the Eq. (6) with the same delay. Hence, continuous dependence on the rhs implies continuous dependence on the delay.

\section{Appendix C}

\section{DDE solvers}

C1 Our solver of choice: dde_solver

The DDE solver dde_solver (Shampine and Thompson, 2006) was used to perform the numerical experiments reported in this paper; dde_solver is a Fortran 90/95 extension of its Fortran 77 predecessor dklag6 (Corwin et al., 1997). Both dde_solver and dklag6 implement a $(5,6)$ pair of continuously embedded, explicit Runge-Kutta-Sarafyan methods. We refer to (Corwin et al., 1997) for the coefficients and precise details of the methods used, and to (Shampine, 1994) for a discussion of continuously embedded Runge-Kutta methods. Both methods in the pair are based on piecewisepolynomial approximants, which are used for error estimation and step size selection, to handle the necessary interpolations for delayed solution values, and to track derivative discontinuities that are propagated by the system delays, while the sixth-order method is used to perform the actual integration.

As discussed in (Shampine and Thompson, 2006), dde_solver was designed to solve systems of DDEs with state-dependent delays in as "user-friendly" a fashion as possible, while at the same time retaining and extending the solution capabilities of dklag6. Our solver was also designed so that usage approaches the convenience of the MATLAB (2007) DDE solvers dde23 and ddesd. For example, storage management is handled automatically by the solver, thus relieving the user of the necessity to supply work arrays whose sizes are not known in advance. Several options are available for supplying necessary information about the problem and for dealing with its special characteristics. All options have carefully chosen defaults that can be changed by the user. These include the ability to supply vectors or functions to define the delays and the initial solution history, the ability to specify points corresponding to known derivative or solution discontinuities, tracking delay-induced derivative discontinuities, the ability to cope with small delays, the ability to handle state-dependent events (e.g., times at which it is 
desirable to make qualitative changes or parameter changes in the underlying system of DDEs), and the ability to solve so-called neutral DDEs, which contain delayed derivatives. The solver builds and returns a Fortran 90 solution structure that may be used for various tasks, e.g., for plotting purposes. An interpolation module uses this structure, for example, to perform additional interpolations requested by the user.

The numerical studies in this paper led us to incorporate several new options in dde_solver. By default, the solver retains the entire solution history. The numerical simulations in this paper, though, require very long integration intervals, leading to prohibitive storage requirements. We added, therefore, an option to have the solver trim points from the solution history queue that precede the largest delay. A related option was added to allow the user to provide a module to process solution information before it is discarded, thus retaining the ability for user interpolation.

The dde_solver with these added options is available at http://www.radford.edu/ thompson/ffddes/. In addition to the solver, a variety of example programs can also be found there; they may be used as convenient templates for other problems. This solver is a Fortran 90/95 compliant selfcontained module with no restrictions on its use. In particular, it is not compiler dependent and has been used successfully with most of the available F90/F95 compilers, including, for example, g95, Lahey LF90, Lahey-Fujitsu LF95, Salford FTN95, SUNf95, and Compaq.

\section{C2 Other DDE solvers}

Other capable DDE software is available. Some noteworthy solvers include archi, ddverk, dde23, and ddesd. Like dde_solver, the first three of these solvers implement pairs of continuously embedded, explicit Runge-Kutta methods. Thus, archi (Paul, 1995) is based on a (4,5) pair, and allows the user to specify either extrapolation or iterative evaluation of implicit formulas. The solver ddverk (Enright and Hayashi, 1997) is based on a $(5,6)$ pair and it handles small and vanishing delays iteratively. Defect error control is used to detect suspected derivative discontinuities, locate them and use special interpolants when stepping over them. The two solvers dde23 (Shampine and Thompson, 2001) and ddesd (Shampine, 2005) are available in the MATLAB problem solving environment; dde23 is based on a $(2,3)$ pair and is applicable to DDEs with constant delays. It is worth noting that the dde 23 user interface led to many of the design decisions used in dde_solver. The solver ddesd incorporates novel methods based on control of the solution residual and is intended for systems with state-dependent delays. In addition to these solvers, two other popular and well-known tools include the DDE-BIFTOOL package (Engelborghs et al., 2001) and the XPPAUT package (Ermentrout, 2002). Each of these packages contains a variety of tools that are useful for analyzing delayed dynamical systems.
Acknowledgements. We are grateful to our colleagues M. Chekroun, J. C. McWilliams, J. D. Neelin and E. Simonnet for many useful discussions and their continuing interest in this work. We want to thank E. Tziperman, two anonymous reviewers, and the Special Issue Editor $\mathrm{H}$. Rust for their valuable comments, queries and suggestions. The study was supported by DOE Grant DE-FG02-07ER64439 from the Climate Change Prediction Program, by NSF Grant ATM-0620838, and by the European Commission's No. 12975 (NEST) project "Extreme Events: Causes and Consequences (E2-C2)."

Edited by: H. Rust

Reviewed by: three anonymous referees

\section{References}

Andronov, A. A. and Pontryagin, L. S.: Systèmes grossiers, Dokl. Akad. Nauk SSSR, 14, 5, 247-250, 1937.

Baker, C. T. H.: Retarded differential equations, J. Comp. Appl. Math., 125, 309-335, 2000.

Baker, C. T. H., Paul, C. A. H., and Willé, D. R.: A bibliography on the numerical solution of delay differential equations, Numerical Analysis Report, 269, Manchester Centre for Computational Mathematics, Manchester, England, 1995.

Battisti, D. S.: The dynamics and thermodynamics of a warming event in a coupled tropical atmosphere/ocean model, J. Atmos. Sci., 45, 2889-2919, 1988.

Battisti, D. S. and Hirst, A. C.: Interannual variability in the tropical atmosphere-ocean system: Influence of the basic state and ocean geometry, J. Atmos. Sci., 46, 1687-1712, 1989.

Bhattacharya, K., Ghil, M., and Vulis, I.: Internal variability of an energy-balance model with delayed albedo effects, J. Atmos. Sci., 39, 1747-1773, 1982.

Bjerknes, J.: Atmospheric teleconnections from the equatorial Pacific, Mon. Weather Rev., 97, 163-172, 1969.

Bodnar, M.: On the differences and similarities of the first-order delay and ordinary differential equations, J. Math. Anal. Appl., 300, 172-188, 2004.

Boulanger, J. P., Menkes, C., and Lengaigne, M. Role of high- and low-frequency winds and wave reflection in the onset, growth and termination of the 1997-1998 El Nino, Clim. Dynam., 22, 2-3, 267-280, 2004.

Cane, M., Munnich, M., and Zebiak, S. E.: A study of self-excited oscillations of the tropical ocean-atmosphere system. Part I: Linear analysis, J. Atmos. Sci., 47, 13, 1562-1577, 1990.

Cao, Y.: Uniqueness of periodic solution for differential delay equations, J. Diff. Eq., 128, 46-57, 1996.

Chang, P., Wang, B., Li, T., and Ji, L.: Interactions between the seasonal cycle and the Southern Oscillation: Frequency entrainment and chaos in intermediate coupled ocean-atmosphere model, Geophys. Res. Lett., 21, 2817-2820, 1994.

Chang, P., Ji, L., Wang, B., and Li, T.: Interactions between the seasonal cycle and El Niño - Southern Oscillation in an intermediate coupled ocean-atmosphere model, J. Atmos. Sci., 52, 2353-2372, 1995.

Chow, S.-N. and Walter, H. O.: Characteristic multipliers and stability of periodic solutions of $\dot{x}=g(x(t-1))$, Trans. Amer. Math Soc., 307, 127-142, 1988. 
Corwin, S. P., Sarafyan, D., and Thompson, S.: DKLAG6: a code based on continuously imbedded sixth order Runge-Kutta methods for the solution of state dependent functional differential equations, Appl. Numer. Math., 24, 319-333, 1997.

Dee, D. and Ghil, M.: Boolean difference equations, I: Formulation and dynamic behavior, SIAM J. Appl. Math., 44, 111-126, 1984.

Delcroix, T., Eldin, G., McPhaden, M., and Morlière, A.: Effects of westerly wind bursts upon the western equatorial Pacific Ocean, February-April 1991, J. Geophys. Res., 98, C9, 16379-16385, 1993.

Diaz, H. F. and Markgraf, V.: El Niño: Historical and Paleoclimatic Aspects of the Southern Oscillation, Cambridge Univ. Press, New York, 1993.

Dijkstra, H. A.: Nonlinear Physical Oceanography: A Dynamical Systems Approach to the Large Scale Ocean Circulation and El Niño, 2nd edition, Springer-Verlag, 2005.

Dijkstra, H. A. and Ghil, M.: Low-frequency variability of the ocean circulation: a dynamical systems approach, Rev. Geophys., 43, RG3002, doi:10.1029/2002RG000122, 2005.

Engelborghs, K., Luzyanina, T., and Samaey, G.: DDE-BIFTOOL v. 2.00: a Matlab package for numerical bifurcation analysis of delay differential equations, Report TW, vd330, Department of Computer Science, K.U. Leuven, Leuven, Belgium, available at http://www.cs.kuleuven.ac.be/cwis/research/ twr/research/software/delay/ddebiftool.shtml, 2005.

Enright, W. H. and Hayashi, H.: A delay differential equation solver based on a continuous Runge-Kutta method with defect control, Numer. Alg., 16, 349-364, 1997.

Ermentrout, B.: Simulating, Analyzing, and Animating Dynamical Systems: A Guide to XPPAUT for Researchers and Students, SIAM, Philadelphia, USA, 2002.

Falbo, C. E.: Analytical and numerical solutions to the delay differential equation $\dot{y}=\alpha y(t-\delta)$, Proc. Joint Mtg. California sections of the MAA, San Luis Obispo, CA, USA, available at: http://www.sonoma.edu/math/faculty/falbo/pag1dde.html, 21 October 1995.

Fuglestvedt, J. S., Berntsen, T. K., Godal, O., Sausen, R., Shine, K. P., and Skodvin, T.: Metrics of climate change: Assessing radiative forcing and emission indices, Climatic Change, 58, 267-331, 2003.

Gebbie, G., Eisenman, I., Wittenberg, A., and Tziperman, E.: Modulation of westerly wind bursts by sea surface temperature: A semistochastic feedback for ENSO, J. Atmos. Sci., 64, 32813295, 2007.

Ghil, M., Allen, M. R., Dettinger, M. D., Ide, K., Kondrashov, D., Mann, M. E., Robertson, A. W., Saunders, A., Tian, Y., Varadi, F., and Yiou, P: Advanced spectral methods for climatic time series, Rev. Geophys., 40, 1, 1003, doi:10.1029/2000RG000092, 2002.

Ghil, M. and Childress, S.: Topics in Geophysical Fluid Dynamics: Atmospheric Dynamics, Dynamo Theory, and Clim. Dynam., Springer, Verlag, 1987.

Ghil, M. and Mullhaupt, A. P.: Boolean delay equations. II: Periodic and aperiodic solutions, J. Stat. Phys., 41, 125-173, 1985.

Ghil, M. and Robertson, A. W.: Solving problems with GCMs: General circulation models and their role in the climate modeling hierarchy, Randall, D.(Ed.) General Circulation Model Development: Past, Present and Future, Academic Press, San Diego, 285-325, 2000
Glantz, M. H., Katz, R. W., and Nicholls, N.: Teleconnections Linking Worldwide Climate Anomalies, Cambridge Univ. Press, New York, 545 pp., 1991.

Hale, J.: Theory of Functional Differential Equations, SpringerVerlag, New-York, 1977.

Harrison, D. E. and Giese, B.: Remote westerly wind forcing of the eastern equatorial Pacific; some model results, Geophys. Res. Lett., 15, 804-807, 1988.

Jiang, N., Neelin, J. D., and Ghil, M.: Quasi-quadrennial and quasibiennial variability in the equatorial Pacific, Clim. Dynam., 12, 101-112, 1995.

Jin, F.-F., Neelin, J. D., and Ghil, M.: El Niño on the Devil's Staircase: Annual subharmonic steps to chaos, Science, 264, 70-72, 1994.

Jin, F.-f., Neelin, J. D., and Ghil, M.: El Niño/Southern Oscillation and the annual cycle: Subharmonic frequency locking and aperiodicity, Physica D, 98, 442-465, 1996.

Katok, A. and Hasselblatt, B.: Introduction to the Modern Theory of Dynamical Systems, Cambridge University Press, 802 pp., 1995.

Kolmanovskii, V. B. and Nosov, V. R.: Stability of Functional Differential Equations, Academic Press, 1986.

Latif, M., Barnett, T. P., Flügel, M., Graham, N. E., Xu, J.-S., and Zebiak, S. E.: A review of ENSO prediction studies, Clim. Dynam., 9, 167-179, 1994.

Lengaigne, M., Guilyardi, E., Boulanger, J. P., et al.: Triggering of El Nino by westerly wind events in a coupled general circulation model Clim. Dynam., 23, 6, 601-620, 2004.

Madden, R. A. and Julian, P. R.: Description of a 40-50 day oscillation in the zonal wind in the tropical Pacific, J. Atmos. Sci., 28, 702-708, 1971.

Madden, R. A. and Julian, P. R.: Description of global-scale circulation cells in the tropics with a $40-50$ day period, J. Atmos. Sci., 29, 1109-1123, 1972.

Madden, R. A. and Julian, P. R.: Observations of the 40-50-day tropical oscillation - A review, Mon. Weather Rev., 122, 5, 814 837, 1994.

Matlab 7.4: The MathWorks, Inc., 3 Apple Hill Dr., Natick, MA 01760, 2007.

Munnich, M., Cane, M., and Zebiak, S. E.: A study of self-excited oscillations of the tropical ocean-atmosphere system. Part II: Nonlinear cases, J. Atmos. Sci., 48, 10, 1238-1248, 1991.

Murphy J. M., Sexton, D. M. H., Barnett, D. N., Jones, G. S., Webb, M. J., and Collins, M.: Quantification of modelling uncertainties in a large ensemble of climate change simulations, Nature, 430, 7001, 768-772, 2004

Neelin, J. D., Latif, M., and Jin, F.-F.: Dynamics of coupled ocean-atmosphere models: the tropical problem, Ann. Rev. Fluid Mech., 26, 617-659, 1994.

Neelin, J. D., Battisti, D. S., Hirst, A. C., Jin, F.-F., Wakata, Y., Yamagata, T., and Zebiak, S.: ENSO Theory, J. Geophys. Res., 103(C7), 14 261-14 290, 1998.

Nussbaum, R. D.: Uniqueness and nonuniqueness for periodic solutions of $\dot{x}(t)=-g(x(t-1))$, J. Diff. Eq., 34, 25-54, 1979.

Nussbaum, R. D.: Functional Differential Equations, available at http://citeseer.ist.psu.edu/437755.html, 1998.

Paul, C. A. H.: A user-guide to ARCHI, Numerical Analysis Report 283, Mathematics Department, University of Manchester, UK, 1995.

Philander, S. G. H.: El Niño, La Niña, and the Southern Oscillation, 
Academic Press, San Diego, 1990.

Saunders, A. and Ghil, M.: A Boolean delay equation model of ENSO variability, Physica D, 160, 54-78, 2001.

Saynisch, J., Kurths, J., and Maraun, D.: A conceptual ENSO model under realistic noise forcing Nonlin. Proc. Geophys., 13, 3, 275285, 2006.

Shampine, L. F.: Numerical Solution of Ordinary Differential Equations, Chapman \& Hall, New York, NY, 1994.

Shampine, L. F.: Solving ODEs and DDEs with residual control, Appl. Numer. Math., 52, 113-127, 2005.

Shampine, L. F. and Thompson, S.: Solving DDEs in Matlab, Appl. Numer. Math., 37, 441-458, 2001.

Shampine, L. F. and Thompson, S.: A friendly Fortran 90 DDE solver, Appl. Num. Math., 56, 2-3, 503-516, 2006.

Stainforth D. A., Aina, T., Christensen, C., et al.: Uncertainty in predictions of the climate response to rising levels of greenhouse gases, Nature, 433, 7024, 403-406, 2005.

Suarez, M. J. and Schopf, P. S.: A delayed action oscillator for ENSO, J. Atmos. Sci, 45, 3283-3287, 1988.

Taylor, K. E.: Summarizing multiple aspects of model performance in a single diagram, J. Geophys. Res., 106, 7183-7192, 2001.
Tziperman, E., Stone, L., Cane, M., and Jarosh, H.: El Niño chaos: Overlapping of resonances between the seasonal cycle and the Pacific ocean-atmosphere oscillator, Science, 264, 72-74, 1994.

Tziperman, E., Cane, M. A., and Zebiak, S. E.: Irregularity and locking to the seasonal cycle in an ENSO prediction model as explained by the quasi-periodicity route to chaos, J. Atmos. Sci., 50, 293-306, 1995.

Verbickas, S.: Westerly wind bursts in the tropical Pacific, Weather, 53, 282-284, 1998.

Yanai, M. and Li, C.: Interannual variability of the Asian summer monsoon and its relationship with ENSO, Eurasian snow cover, and heating, Proc. Int. Conf. on Monsoon Variability and Prediction, Trieste, Italy, World Meteorological Organization, 27-34, 9-13 May 1994.

Zaliapin, I., Keilis-Borok, V., and Ghil, M.: A Boolean delay equation model of colliding cascades. Part I: Multiple seismic regimes, J. Stat. Phys., 111, 815-837, 2003.

Zebiak, S. and Cane, M. A.: A model El-Niño Southern Oscillation, Mon. Weather Rev., 115, 2262-2278, 1987. 